OPEN ACCESS

Edited by:

Paul Roesch,

University of Bayreuth, Germany

Reviewed by:

Margarida Simões,

University of Evora, Portugal

Fernando Costa Ferreira,

University of Lisbon, Portugal

*Correspondence:

Dan $\mathrm{Li}$

lidan@caas.cn

Haixue Zheng

haixuezheng@163.com

Specialty section:

This article was submitted to

Viral Immunology,

a section of the journal

Frontiers in Immunology

Received: 14 June 2021

Accepted: 20 August 2021

Published: 06 September 2021

Citation:

Wang $Y$, Kang W, Yang W, Zhang J, Li $D$ and Zheng $H$ (2021) Structure of African Swine Fever Virus and Associated Molecular Mechanisms Underlying Infection and Immunosuppression: A Review.

Front. Immunol. 12:715582. doi: 10.3389/fimmu.2021.715582

\section{Structure of African Swine Fever Virus and Associated Molecular Mechanisms Underlying Infection and Immunosuppression: A Review}

\author{
Yue Wang, Weifang Kang, Wenping Yang, Jing Zhang, Dan $L i{ }^{*}$ and Haixue Zheng * \\ State Key Laboratory of Veterinary Etiological Biology and OIE/National Foot and Mouth Disease Reference Laboratory, \\ Lanzhou Veterinary Research Institute, Chinese Academy of Agricultural Sciences, Lanzhou, China
}

African swine fever (ASF) is an acute, highly contagious, and deadly infectious disease. The mortality rate of the most acute and acute ASF infection is almost 100\%. The World Organization for Animal Health [Office International des épizooties (OIE)] lists it as a legally reported animal disease and China lists it as class I animal epidemic. Since the first diagnosed ASF case in China on August 3, 2018, it has caused huge economic losses to animal husbandry. ASF is caused by the African swine fever virus (ASFV), which is the only member of Asfarviridae family. ASFV is and the only insect-borne DNA virus belonging to the Nucleocytoplasmic Large DNA Viruses (NCLDV) family with an icosahedral structure and an envelope. Till date, there are still no effective vaccines or antiviral drugs for the prevention or treatment of ASF. The complex viral genome and its sophisticated ability to regulate the host immune response may be the reason for the difficulty in developing an effective vaccine. This review summarizes the recent findings on ASFV structure, the molecular mechanism of ASFV infection and immunosuppression, and ASFV-encoded proteins to provide comprehensive proteomic information for basic research on ASFV. In addition, it also analyzes the results of previous studies and speculations on the molecular mechanism of ASFV infection, which aids the study of the mechanism of clinical pathological phenomena, and provides a possible direction for an intensive study of ASFV infection mechanism. By summarizing the findings on molecular mechanism of ASFV- regulated host cell immune response, this review provides orientations and ideas for fundamental research on ASFV and provides a theoretical basis for the development of protective vaccines against ASFV.

Keywords: African swine fever virus, immunosuppression, nucleocytoplasmic large DNA virus, infection, animal disease

\section{INTRODUCTION}

African swine fever virus (ASFV) is a $200 \mathrm{~nm}$ diameter icosahedral DNA virus comprising envelope, capsid, inner capsule membrane, core shell, and inner core. The viral genome is a linear 170-190 kb long double-stranded DNA molecule with covalently closed ends. The size of the DNA is 170kb-190kb depending on the virus strain and encodes 150-200 viral proteins, including 68 structural proteins and more than 100 non-structural proteins (1-4). The repeat and loss of certain sequences in the ASFV genome is one of the factors for differences in ASFV strains from different sources or different 
generations of the same strain (5). P72 is the major capsid protein, which is used for serotyping of ASFV strains because of its conservativeness $(6,7)$. Monocyte-macrophages are the main target cells of ASFV (7). The molecular mechanism of ASFV infection in host cells is still unclear. The clathrin-dependent endocytic pathway and the macropinocytosis pathway are the probable pathways for ASFV invasion. However, the cell membrane receptors and viral proteins mediating this process remain unknown. The internalized virus particles rely on the of the host cell endosomal system to move from the edge of the membrane to the center. With the gradual acidification of the endosome, ASFV removes the outer shell and inner envelope, releasing the viral genome into the cytoplasm (8). There are two stages of ASFV replication. The first stage of replication takes place briefly in the nucleus and then a large number of DNA fragments are synthesized in the virus factory (VF) in the perinuclear region (9). Viral gene expression is divided into four stages: immediateearly, early, middle and late (10). ASFV has a set of hostindependent replication and transcription mechanisms, but the translation process remains host-dependent $(2,11)$. Genome replication and transcription rely on many ASFV genomeencoded related proteins (5). ASFV also performs posttranscriptional and post-translational modifications, such as 5'mRNA capping of mRNA and protein acetylation of proteins, which are favorable for intracellular viral genome expression (12, 13). The synthesized virus proteins assemble at near the VF, holding the endosomal system to transport the progeny virus to cell membrane, and then bud and release.

The viruses are identified and attacked by the host immune system. For the benefit of reproduction, the viruses have evolved several mechanisms to evade and suppress immune response. Innate immunity is the first line of defense, which is also one of the targets of viral immunosuppression. ASFV is mainly recognized by cGAS, which then transmits signals downstream by signaling pathways to produce an antiviral response. Thus, ASFV weakens the immune response by blocking signal delivery with multiple molecules on the antagonized cGAS/STING pathway (14-16). In addition, to a certain extent, cells inhibit virus spread by initiating their apoptosis procedures. Accordingly, ASFV encodes various viral proteins that inhibit both the exogenous and endogenous apoptosis pathways in the early stage (14). ASFV primarily regulates cytokines such as interferon (IFN) and tumor necrosis factor alpha (TNF- $\alpha$ ). ASFV not only restrains IFN and TNF- $\alpha$ activation, transcription and expression, but also inhibits the expression of their downstream proteins (16). In addition, ASFV inhibits adaptive immune responses, such as major histocompatibility complex (MHC)-I/II expression and cytotoxic T-cell activation (14, 16, 17). The transcription factor nuclear factor kappa beta (NF- $\kappa \mathrm{B})$ induces proinflammatory factor expression. Therefore, ASFV can regulate inflammatory response by regulating NF- $\mathrm{BB}$.

\section{STRUCTURE}

ASFV is a giant icosahedral DNA virus. The diameter of the virus particles is between $260-300 \mathrm{~nm}$. ASFV has a multiple-layer structure, including the envelope, capsid, inner envelope, core shell and nucleoid from outside to inside $(18,19)$ as shown in Figure 1.

\section{The Outer Envelope (p12/pE402R)}

The outer envelope is the outermost layer of ASFV which is acquired it from the host cellular membrane during budding. Some fractions of protein pEP402R (CD2v) have been detected on the outer layer of budding virions. The viral pE402R homolog is the only marker molecule of the outside-virus structure (1). p12 promotes the adsorption of virus particles on host cells as an outer envelope protein by binding to specific receptors on the host cell membrane to mediate ASFV entry. However, other studies have shown that $\mathrm{p} 12$ is localized on the inner envelope of the virus using immunoelectron microscopy $(1,20)$.

\section{The Capsid (pE120R/pB438L/P72)}

The diameter of the largest ASFV capsid is approximately $250 \mathrm{~nm}$. The capsid components are 2,760 pseudo-hexameric capsomers and 12 pentameric capsomers. Every three p72 protein molecules adopting a double jelly-roll structure forms one pseudo-hexameric capsomer, and another five penton proteins can construct a pentameric capsomer (19). Protein pB438L is necessary for the capsid to form its vertices. In addition to proteins $\mathrm{p} 72$ and pB438L, pE120R also belongs to the virus capsid (1).

\section{The Inner Envelope (p12/p17/pE183L/ pE248R/pH108R/pE199L/P22)}

The third layer, the inner envelope, is a 70 - $\AA$ thick lipid bilayer membrane divided from the endoplasmic reticulum (ER) (2). A previous study has pointed out that $\mathrm{pE} 183 \mathrm{R}$ is a key protein involved in inner envelope formation. A recent study has reported the presence of p17, pE183L, p12, pE248R, and pH108R in the inner envelope using immunoelectron microscopy. p17 and pE183L primarily help to assemble the capsid layer, while p12, pE248R, and pE199L are involved in virus entry. pE248R, and pE199L are believed to be a part of the virus integration mechanism. In addition, some researchers have suggested that the p22 protein is also a component of the inner viral membrane (1).

\section{The Core Shell (p5/p14/p34/p37/p150/p15/ p35/p8/pS273R)}

The fourth layer is a $180-\mathrm{nm}$-diameter, called the core shell. Two kinds of virus polyprotein precursors, pp220 and pp62, are broken down into many mature products through viral protease (pS273R) to form the core shell. P150, p37, p34, p14 and $\mathrm{p} 5$ are developed from pp220, while p35, p15, and p8 are developed from pp62 $(1,2)$.

\section{The Nucleoid (p10/pA104R)}

The innermost of virus particles are nucleoids. ASFV genome is approximately 170-194 kbp linear double-stranded DNA and encodes $150-170$ open reading frames (10). The terminal of the viral genome consists of covalently cross-linked hairpin loops (2). p10 and pA104R are DNA binding proteins that have been detected in the viral nucleoid (1). Besides, the ASFV genome codes many non-structural proteins related to virus replication, transcription, and immunosuppression (Figure 1). 


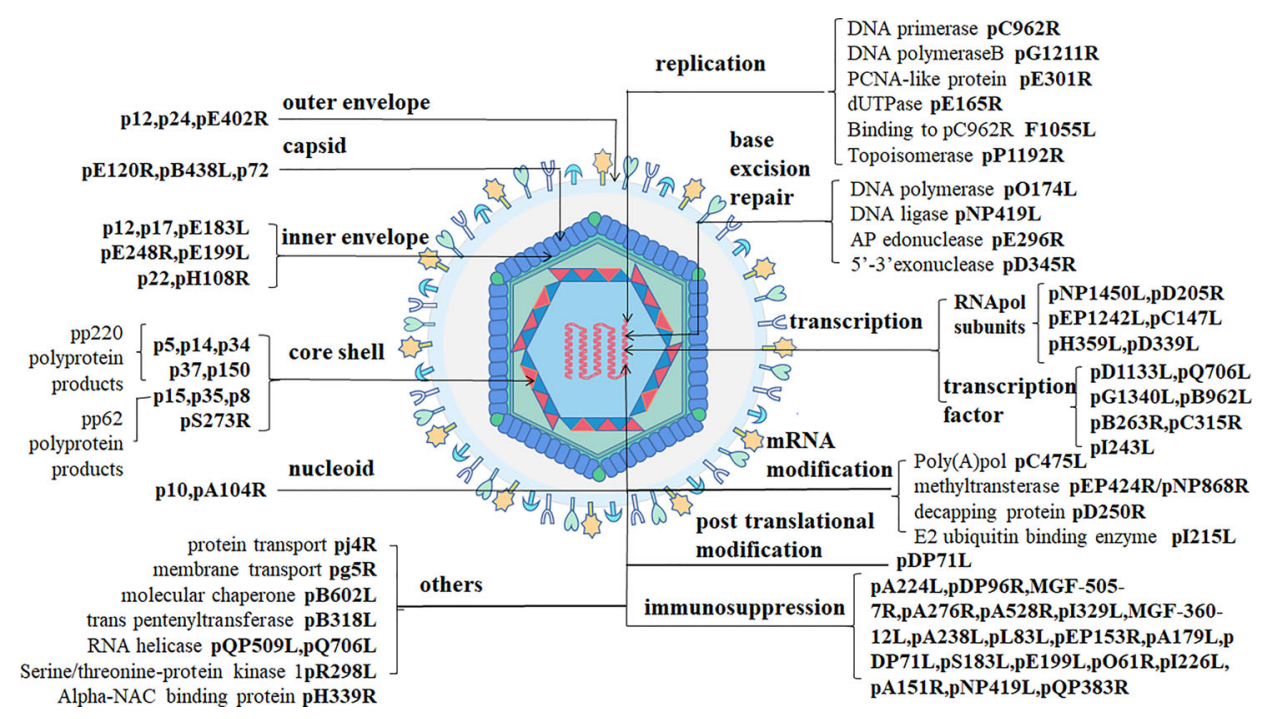

FIGURE 1 | ASFV structure (1). ASFV-encoded structural proteins constitute the envelope (p12/p24/pE402R), the capsid (pE120R/pB438L/p72), and the inner envelope (p12/p17/pE183L/pE248R/pH108R/pE199L/p22). p5, p14, p34, p37, and p150 are pp220 hydrolysates produced by pS273R. p15, p35, and p8 are p62 hydrolysates. DNA-binding proteins include pA104R and p10. ASFV-encoded proteins with clear functions include those involved in viral DNA replication (pC962R/ pG1211R/pE301R/pE165RF1055L/pP1192R), base excision repair (p0174L/pNP419L/pE296R/pD345R), transcription (pNP1450L/pD205R/pEP1242L/pC147L/ pH359L/pD339L/pD1133L/pQ706L/pG1340L/pB962L/pB263R/pC315R/pl243L), mRNA modification (pC475L/pEP424R/pNP868R/pD250R/pl215L), post-translational modification (pDP71L), immunosuppression (pA224L/pDP96R/MGF-505-7R/pA276R/pA528R/pl329L/MGF-360-12L/pA238L/pL83L/pEP153R/pA179L/pDP71L/ pS183L/pE199L/p061R/pl226L/pA151R/pNP419L/pQP383R), and other proteins (pj4R/pg5R/pB602L/pB318L/pQP509L/pQ706LpR298I/pH339R).

\section{ASFV INFECTION MECHANISMS}

To infect host cells successfully, viruses must undergo six events including adsorption, penetration, uncoating, biosynthesis, packaging, and shedding. ASFV is internalized within 30mip after binding to the host receptor or through macropinocytosis pathway. Virion gets into early endosomes during 1-30 minutes post-infection (mpi) and is transported into late endosomes between 30 to $90 \mathrm{mpi}$. After that, ASFV completed the shelling and genome release in the late endosomes. Gene expression can be divided into three stages: early, intermediate and late gene expression. Early genes expression occurs at 4-6 hours post-infection (hpi) and mainly encode ASFV replication related proteins, followed by the replication of ASFV genome at 6-8 hpi. Genome replication occurs first in the nucleus, mainly in the cytoplasm. After replication, the intermediate and late genes which encode virus particle structure related proteins start expression at 8-16 hpi. At 16-24 hpi, virus particles are assembling at virus factory. About $24 \mathrm{hpi}$, the assembled virus particles sprout from the cell membrane to release outside (7).

This study describes the mechanism of ASFV infection through ASFV entry, transport, genome duplication, transcription, translation, VF formation, and offspring-virion assembly and release. Monocyte-macrophages are the main host cells of ASFV. Moreover, ASFV also infects secondary target cells such as vascular endothelial cells, hepatocytes or epithelial cells (21).

\section{ASFV Invasion Mechanism}

Previous studies have shown that ASFV entry into host cells, a receptor-mediated endocytosis process, is temperature-, energy-, cholesterol-, and low-pH-dependent. Recent studies have described this process to be clathrin-dependent endocytosis (CME) and macropinocytosis. Both $\mathrm{CME}$ and macropinocytosis require dynamin. The obvious negative effect of inhibition key proteins needed for ASFV entry by several pharmacological inhibitors and specific proteins indicates that the main pathway for entry is macropinocytosis. Macropinocytosis depends on actin rearrangement and also involves the cholesterol, sodium/proton exchanger (Na/H), EGFR, PKC, phosphorylated PI3K, Pak1 kinase, and small Rho-GTPase Racl activation $(2,21)$ (Figure 2). However, in some studies, purified viral particles did not cause significant macropinocytosis events in Vero cells and macrophages (25). Interestingly, clinical symptoms suggest that ASFV-infected macrophages show antibody-dependent enhancement (ADE). In other words, the $\mathrm{Fc}$ receptor may participate in this process, but further studies needed to verify this hypothesis (7). Taken together, it is easy to speculate that ASFV prefers utilizing different pathways to improve its ability to infect various target cells and adapt to the changing conditions of the infection process.

\section{Adsorption}

CD163 antibody inhibits ASFV infection in macrophages, which indicates that the $\mathrm{CD} 163$ receptor on the surface of macrophages is closely related to ASFV susceptibility. Nevertheless, the mortality, pathological anatomy, and viremia of ASFV-infected CD163 gene knockout pigs were not significantly different from those in the control pigs. This result suggests that CD163 alone is not enough for virus particles to infect host cells and other proteins are also involved in this process (9). In addition, CD45 


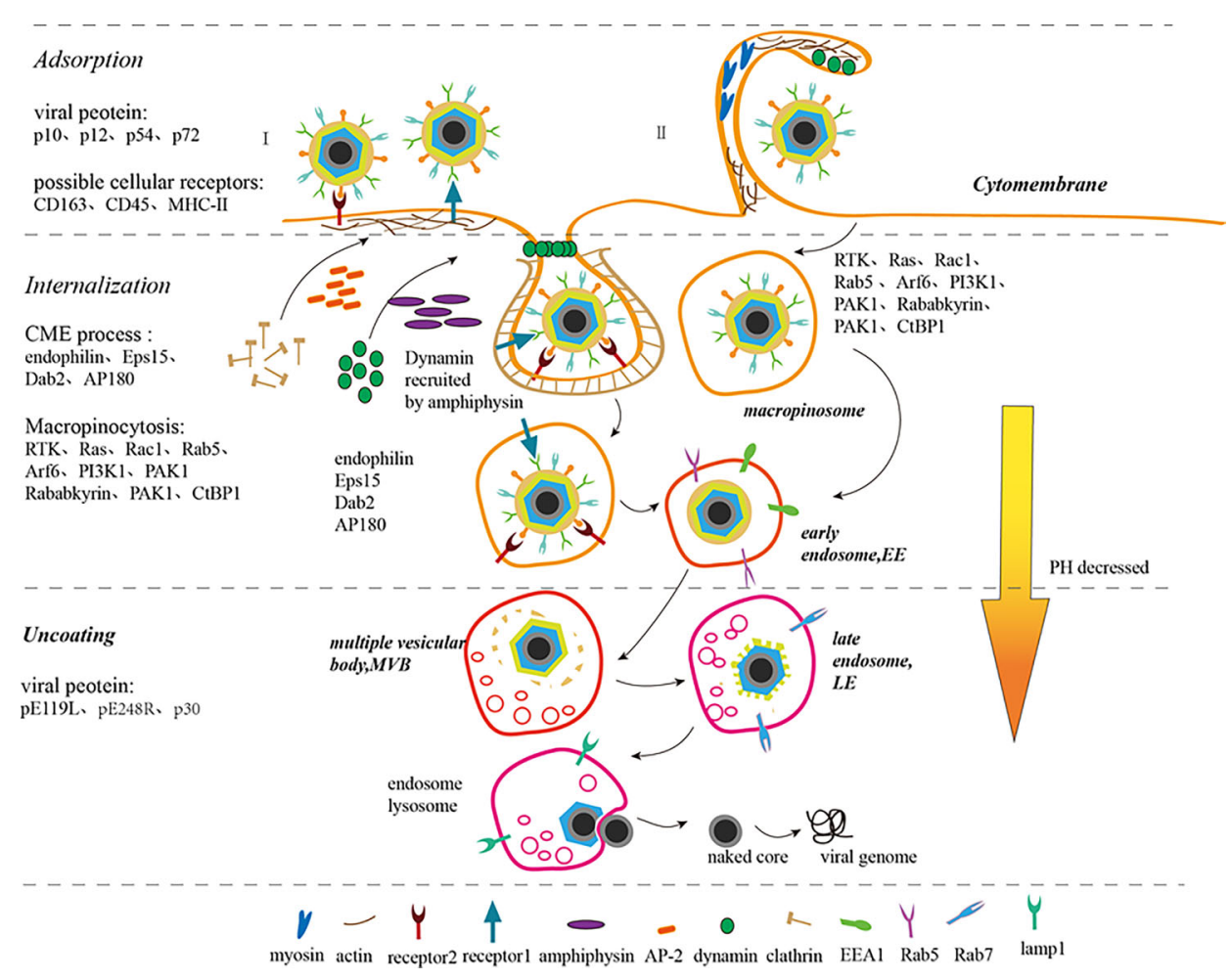

FIGURE 2 | ASFV adsorption, internalization, uncoating, and release process (7-9, 21-24). ASFV enters the host cell through endocytosis (I) and macropinocytosis (II). In endocytosis (I), the virus binds to the cell receptors to form coated pits. Adaptor protein-2 (AP-2) recruits clathrin to accumulate and assemble in coated pits and amphiphysin recruits dynamin simultaneously. Disabled protein 2 (Dab2), AP180, and Eps15-interacting protein (EPsin) can also recruit clathrin. The coated pits sink downward under the action of dynamin, endophilin, and actin. Simultaneously, dynamins assemble on the depressed neck and then cut the depressed corpuscles to separate it from the cell membrane. The separated small vesicles lose clathrin, thus forming early endosomes (EE). In micropinocytosis (II), the virions induce and activate RTK, Ras, Rac1, Rab5, Arf6, and PI3K1 signals, which activate actin, thus rearranging actin and microfilaments to form ruffles or blebs on the cell membrane surface. PAK1 and Arf6 regulate the dynamic cytoskeletal changes and induce membrane bending. Rab5, Rababkyrin, PAK1 and CtBP1 induce ruffle closure. Myosin provides contractile activity for ruffle closure. The macropinosomes are equivalent to the EEs of the cell. EEs migrate, mature and are acidified within the cell to form multiple vesicular bodies (MVBs), late endosome (LE), and endosome lysosome gradually. ASFV sheds its capsid in LE, fuses its inner capsule with the restricted endosome membrane, releases its core shell into the cytoplasm, and then releases its DNA.

and MHC-II play a role in ASFV invasion of macrophages (26). In short, the cell receptors mediating ASFV adsorption on host cell surface are still unknown.

Till date, studies have shown that p10, p12, p54, and p72 mediate ASFV adsorption. p10 not only binds to single-stranded and double-stranded DNA, but also enters the nucleus $(8,27)$. p54 and p72 neutralizing antibodies can blocks the ASFV adsorption to macrophages, indicating that $\mathrm{p} 54$ and $\mathrm{p} 72$ proteins are involved in ASFV adsorption (7). The ASFV EP402R protein mediates adsorption on red blood cell surface harboring a certain similarity with CD2v located on the cell surface (28).

\section{Internalization}

In the CME process, virions bind to specific receptors firstly on the cell membrane, and then form coated pits at specific sites. Adaptor protein-2 (AP-2) specifies the clathrin assembly site on the lipid membrane and promotes clathrin aggregation and assembly on the plasma membrane. Disabled protein 2 (Dab2) also exhibits clathrin assembly activity. Both AP180 and Eps15interacting protein (Epsin) accelerate clathrin assembly in vitro.
Dynamin binds to GDT in the presence of amphiphysin and locates on the cell membrane through interaction with PtdIns(4,5)P2 via its $\mathrm{PH}$ domain. The coated pits on the plasma membrane invaginate under the action of dynamin, endophilin and actin, and then the clathrin-formed sunken cell membrane encloses the virus particles together with cell receptors. The GDT/GTP exchange allows dynamin to fall off the coated pits and concentrate in the depressed neck. Then, the depressed corpuscles are cut by dynamin to separate them from the cell membrane. The isolated vesicles lose clathrin and form early endosomes $(\mathrm{EE})(8,22)$. Related studies have shown that clathrin-coated pit formation will be inhibited in Eps15-deficient Vero or WSL cells. Cholesterol is also essential for clathrin-coated vesicle formation (29).

Macropinocytosis is a non-selective endocytosis process. During macropinocytosis, virus particles induce RTK and Ras activity which then activate downstream molecules, such as Rac1, Rab5, Arf6, and PI3K1, causing actin and microfilaments rearrangement to form ruffles or blebs on the cell membrane surface. While virions are adsorbed on the cell membrane surface, PAK1 regulates the dynamic changes of the cytoskeleton and Arf6 
induces membrane curvature to wrap the virus particles in the raised folds. Subsequently, Rab5 with its effector molecules Rababkyrin, PAK1 and CtBP1 induce ruffle closure, while simultaneously providing contractile activity for the closure of endocytic vesicles. The formation of macropinosomes, corresponding to early endosomes (EEs), shows that the virus has successfully completed internalization $(8,23)$. Viruses that enter cells through this process include vaccinia virus, adenovirus, and picornavirus. The outer envelope of mature virus particles is rich in phosphatidylserine, which is a phospholipid necessary for cells to absorb cell debris through macropinocytosis. Changing the lipid content of the outer membrane of the virus can result in failure of the internalization into cells, which provides a strong evidence that the virus particles induce the macropinocytosis in cells by simulating apoptosis (23). However, some researchers believe that ASFV does not specifically activate the process of micropinocytosis because macropinocytosis can also be produced by the intake of many nutrients and removal of apoptotic cell debris (8).

The two methods of viral internalization are not antagonistic, but cooperate with each other, for instance, actin rearrangement is also involved in the CME process.

\section{Uncoating}

EE migrates and matures within the cell, transforming into acidic multiple vesicular bodies (MVBs), late endosomes (LEs), or endosomal lysosomes fused with lysosomes. In LE, the ASFV completely uncoats in an acidic environment. The uncoating of the virus requires acidic conditions, and shelling is inhibited if acidification of endosomes is prevented (8). Factors affecting virus uncapsidation also include the cholesterol entry through the endocytic pathway and the regulation of the ubiquitinproteasome of host cells. Inhibiting the ubiquitin-proteasome pathway prevents ASFV decapsidation, suggesting that ubiquitin-proteasome is also essential for ASFV uncoating. The ubiquitin-proteasome system is required for ASFV replication $(30,31)$. Then, the inner envelope membrane fuses with the restricted endosome membrane and the viral DNA is set free from the core shell following the release of the core shell into the cytoplasm (8). Vaccina virus (VACV), which also belongs to the NCLDV family, releases virions from endosomes to the cytoplasm through an entry fusion complex (EFC) composed of 11 viral polypeptides. Interestingly, the ASFV-encoded protein pE199L encoded by ASFV is thought to be essential for fusion of the inner membrane with the restrictive endosome membrane and the release of the core shell because of its sequence similarity with the components of VACV EFC including G9, A16, and J5 proteins (32). The sequence and structural characteristics of pE248R, also located in the inner capsule membrane of ASFV, are identical to those of the L1 protein, which is also one of the compositions of EFC. Therefore, it is supposed that ASFV may have a fusion mechanism composed of pE199L and pE248R, which is a simplified version of the VACV EFC. Homologous EFCs have also been found in other viruses in the NCLDV family, such as iridoviruses and phycodnaviruses $(21,32)$. Furthermore, another study has reported that the protein pE248R located in the inner envelope membrane mediates the fusion of the viral inner membrane and the restricted endosome membrane with the cholesterol present in the endosomal membrane. This phenomenon validates the speculation that ASFV has a similar structure of EFC. In addition, the process of ASFV DNA release from core shell to the cytoplasm is supposed to be similar to that of other viruses depending on the host cell's ubiquitin-proteasome pathway (21). Other studies have shown that the release of virus particles is also impeded by microtubule inhibition indicating that microtubules play an important role in ASFV invasion (8). The structural protein P30, an early protein encoded by ASFV, is also believed to play a significant role in virus internalization (7).

\section{ASFV Traffic Mechanism in Host Cells}

The transport of virions from the EE to the VF and then the cell membrane depends on the function of microtubules (33). After the virion is internalized, the vesicles successively form early endosome (EE), mature endosome (ME), and late endosome (LE) in the cell (24). As EEs containing ASFV mature into LEs, the endosome cavity acidizes steadily, the molecular markers on the endosomal membrane surface gradually change, and finally the virus particles are unshelled in an acid environment. ASFV infection redistributes the entire vesicle system so that virus particles can be easily transported to the perinuclear region (3). Immediately after infection (5-30 mpi), capsid and inner envelope proteins co-localize with specific markers such as EEA1 and Rab5 in EE and macropinocytosome. In the late-stage (30-90 mpi), the viral inner envelope protein (P17) or core shell protein (P150) co-localize with the late endosomal marker (Rab7), a key regulator of ASFV infection, and the lysosome marker (Lamp1) (21, 33). After the virus particles are assembled in the VF, the kinesin uses a multi-terminal microtubule motor to transport the "cargo" from the VF to the cell membrane.

Intriguingly, ASFV may also increase microtubule stability by over-acetylating microtubules to ensure that mature virus particles are transported to the plasma membrane. The actin cytoskeleton is considered to be a possible alternative route for retrograde viral transport (34).

\section{ASFV Replication and Gene Expression Mechanism in Host Cells}

ASFV, released from the endosome, migrate to the perinuclear microtubule tissue through activating the microtubule system by the viral inner envelope protein pE183L (p54) combined with the microtubule dynein for the DNA replication and biosynthesis (9, 35, 36) (Figures 3-5). Viral gene expression is divided into four stages: immediate, early, intermediate, and late stage. Early genes are expressed within 4-6 h of virus infection, including proteins and enzymes necessary for viral genome replication, required for late gene expression and multi-gene families related to immune evasion. After ASFV infection for approximately 6-8 h, the virus begins to replicate. Although replication of ASFV mainly occurs in the VF in the cytoplasm, there is also a brief moment in the nucleus for duplication in the early stage. After 8-16 h, mid- and late-stage genes begin to express to synthesize structural proteins required to form nascent virus and early transcription factors packaged in the virus particles $(7,37)$. Studies have shown that 


\section{Replication}

\section{viral protein:} pC962R pG1211R pE301R pE165R,F1055L,pP1192R pA104R,p10,p14p37 pP1192R

BER:

pO174L、pNP419L

pE296R、pD345R

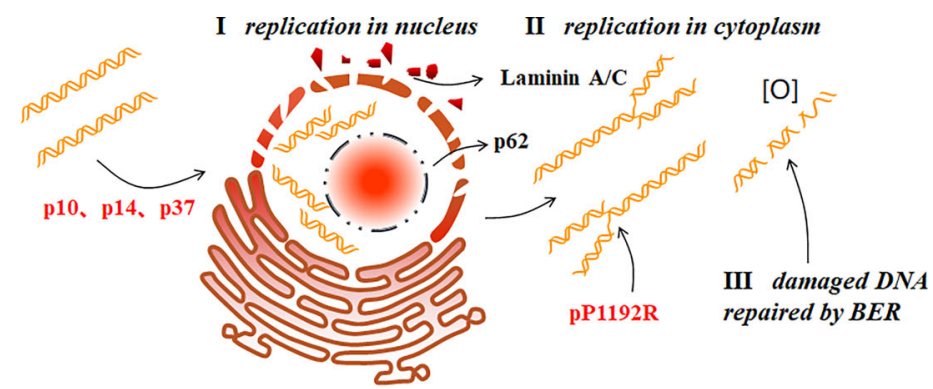

FIGURE 3 | ASFV replication (9). ASFV first replicates briefly in the nucleus (I), and then the cytoplasm (II). p37 mediates the nucleocytoplasmic transport of ASFV genome. In addition, p10 and p14 can also enter the nucleus. ASFV replication in the nucleus ruptures the nucleolus and nuclear membrane, thus collapsing laminin AVC and recruiting the nucleoporin p62 to the periphery. ASFV produces short DNA fragments the nucleus and long fragments in the cytoplasm. pP1192R catalyzes transient nick generation in double-stranded DNA and promotes the translation process. To prevent active oxygen-mediated damaged DNA from interfering with replication, ASFV also uses BER to repair the damaged DNA (III).

there are 68 types of viral proteins through mass spectrometry analysis, including structural proteins and non-structural, which are indispensable for virus transcription, DNA repair, and protein modification (38).

\section{Transcription}

ASFV encodes POLV subunits and the TATA-binding protein (TBP)-like protein (B263R). ASFV C315R encodes a TFIIB-like factor, and $\mathrm{I} 243 \mathrm{~L}$ encodes a transcription splicing/elongation factor TFIIS homolog $(37,40)$. Based on the above studies, we can assume that ASFV has a host-independent transcription system which is similar to the eukaryotic POLt transcription mechanism. In the POLII transcription mechanism in eukaryotic cells, TBP binds to the TATA box, and then TFIIB binds to the TFIIB recognition element (BRE). Finally, RNA Pol and related general transcription factors (GTFs) are recruited to form the pre-transcription initiation complex (PIC). The energy generated by ATP hydrolysis promotes transcription initiation. Factors promoting transcription elongation include TFIIS and SPT5. Hence, it has been suggested that ASFV can also forms a similar PIC. Recent studies have shown through sequencing that $\mathrm{TA}^{*}$ and TA ${ }^{\star}$ TA motifs exist at the initiator (Inr) upstream of the TSS in the early and late promoter sequences respectively, and a conservative early promoter motif (EPM) and late promoter motif (LPM) have been found in the region further upstream of the TSS. The EPM is similar to the upstream control element (UCE) of the VACV. UCE binds to the transcription initiation factor heterodimer composed of D6 and D7. VACV and ASFV both belong to the NCLDV family. Therefore, from the transcription mechanism of $\mathrm{VACV}$, it can be inferred that the ASFV- encoded D6/D7-like factor can binds to the EPM upstream of the early gene, inducing early promoter transcription. The virus-encoded TBP/TFIIB homolog expressed in the early stage of infection, is recruited to the LPM upstream of the late gene and promotes late or post-replication transcription. However, apart from the same T/A-rich nature and its location at the upstream of the TSS, LPM has no obvious similarity to the TATA box (37). Through TSS analysis, it has been found that ASFV increases protein diversity and retention by using alternative TSS, thereby synthesizing mRNA shortened at the 5'-terminal and encoding $\mathrm{N}$ terminal truncated proteins. Some studies have also shown that the transcription mechanisms of ASFV and VACV are host -independent and similar to the transcription of certain yeast plasmid-encoded toxin genes. These results suggest that the NCLDV family and the killer plasmids share a common evolutionary pathway (37). While studying ASFV transcription termination by the third NGS, a polyU termination motif has been detected in the RNA of approximately $2 / 3$ of early and late genes, but not in nearly $1 / 3$ of the RNA. Therefore, ASFV transcription termination may be factor-dependent combined with the conservative evolution of VACV RNA helicase.

After transcribing, the pre-RNA undergoes 3 '-polyadenylation and 5'-capping in the cytoplasm. Compared with VACV, it is conjectured that adding the PolyA tail to the $3^{\prime}$-terminal of the transcript involves the participation of an ASFV-encoding polyadenylation enzyme (C475L) (5). Three enzymes are involved in 5'-capping: 5'triphosphatase (TPase), guanylate transferase (GTase) and methyltransferase (MTase). ASFV PNP868R ${ }^{\mathrm{MT}}$ a type I MTase folds according to its structure analysis which remained significantly similar to cap MTase in many cells and viruses. The high conservation of key residues indicates that the recognition characteristics of PNP868R ${ }^{\mathrm{MT}}$ cap are similar to those of the host cell and virus particles (12). Another in vitro study has reported that Ba71V D250R encodes a decapping protein (ASFV$\mathrm{DP})$, which interacts with ribosomal protein L23a and the mRNA cap structure located on the ER and also interacts with poly(A) RNA through the N-terminal structure resulting in a decrease in the number of transcripts of the virus and host cells to shut off the cell and regulate the time of viral gene expression (41).

\section{Translation}

ASFV proteins synthesis depends on the host translation mechanism of the host cell. ASFV hijacks eukaryotic initiation factors (eIFs), including eIF2, eIF4F, eIF4G and eIF4E (41) (Figure 5). Moreover, ASFV I226R and I243L are expressed in multiple independent promoter-controlled periods (7). Interestingly, most post-transcriptional ASFV RNAs contains AU and AUAU-leaders (37). Whether they improve translation 


\section{Transcription}

viral protein: pNP1450L,pD205R,pEP1242L pC147L,pH359L,pD339L pD1133L,pQ706L,pG1340L pB962L,pB263R,pC315R pI243L,pI215

post transcriptional modification: pC475L,pEP424R,pNP868R pD250R

post transcriptional modification

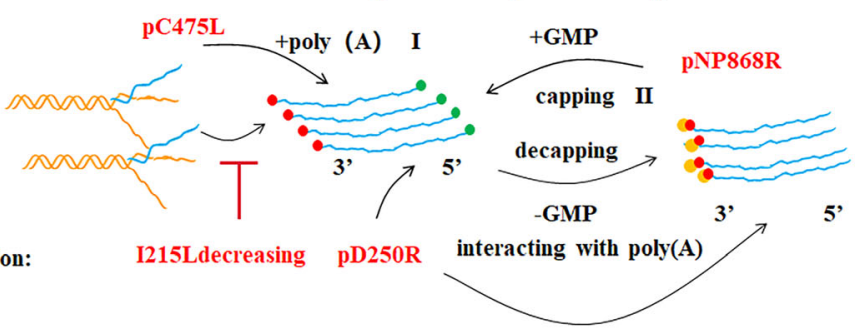

poly(A)

FIGURE 4 | ASFV transcription and post-transcriptional modification (39). Pre-mRNA obtained after ASFV transcription is modified in the cytoplasm by capping the 5' end (II) and poly adenylating the 3' end (I). ASFV encodes a polyadenosine enzyme (pC475L) that may be involved in 3' end modification. ASFV pNP868R participates in 5' end modification. Ba71V D250R encodes a decapping protein (ASFV-DP), which could interact with poly(A) of the 3' end.

efficiency like 5 '-RNA poly(A) leaders in eukaryotic cells and VACV is still unknown. Researchers have found that the E2 ubiquitin-conjugating enzyme (I215L) is an early expression gene. I215L expression decrease directly leads to B646L transcript downregulation. It has been speculated that $\mathrm{I} 215 \mathrm{~L}$ participates in multiple events in the ASFV life cycle based on the monoubiquitination, diubiquitination, and polyubiquitination function of I215L $(3,42,43)$. Proteins related to the core shell and proteins involved in ASFV DNA replication may be targeted by ubiquitin-proteasome $(3,30)$. These studies indicate that viral replication and late gene expression are mediated by the ubiquitin pathway.

ASFV regulates the DNA translation pathway through multiple methods. For example, the virus protein DP71L binds to hostencoded phosphatase 1 (PP1) and dephosphorylates eIF2 $\alpha$ to enhance viral protein synthesis $(44,45)$. ASFV protein A224L, an IAP homolog, also increases viral protein synthesis by inhibiting caspase-3-mediated eIF4G degradation in the eIF4F complex (eIF4A, eIF4E, and eIF4G) $(46,47)$. ASFV can stimulate mTORmediated serine108 phosphorylation on eIF4G and Mnk-1mediated serine209 phosphorylation on eIF4E to enhance viral protein products (47). ASFV promotes 4E-BP phosphorylation to enhance viral protein translation in the early stage of infection, while promotes $4 \mathrm{E}-\mathrm{BP}$ dephosphorylation to inhibit translation in the late-stage (2). ASFV also increases the quantity of translation component eIFs remaining in the viral VF, which promotes viral protein synthesis while inhibiting host protein synthesis (3). ASFV also has the ability of making histone $\mathrm{H} 3 \mathrm{~K} 9 / \mathrm{K} 14$ of host cell in a low-acylation state to promote viral gene expression (13). In addition, some researchers discovered that EP424R-encoded FTS-J-like RNA methyltransferase may stabilize rRNA in cells to prevent protein synthesis machine shutdown (5).

\section{Replication}

The nucleus is the starting site for ASFV DNA replication which then diverts into the cytoplasmic (7). The presence of the nucleus is essential for the early ASFV DNA synthesis (48). C962R of ASFV encodes a DNA primerase similar to that encoded by VACV (D5). This primerase may function like D5 which plays a role in DNA replication initiation and modifies the DNA replication mode. Thus, a new pattern similar to that of VACV replication initiation has been proposed: replication is initiated by introducing a single-stranded gap near one or both ends of the genome. The exposed $3^{`}-\mathrm{OH}$ acts as a primer for DNA polymerase when the DNA is synthesized from the end of the genome. This creates a "head-to-head concatemers" where the ends of the nascent and template strands are self-complementary and folded forming a self-starting hairpin structure to initiate DNA replication. The ASFV DNA fragments produced in the nucleus are short fragments that form long fragments or genomes with large molecular weights in the cytoplasm, but not in the nucleus. Next, large DNA fragments in the cytoplasm gradually develop into mature cross-linked DNA (5). p37 is involved in the nucleocytoplasmic viral DNA transport during ASFV replication. In the early stage of infection, p37 accompanies viral DNA entry and accumulation in the nucleus from the cytoplasm. After replication in the nucleus, they are transported to the cytoplasm and accumulate in the VF. Nuclear export protein 1 (CRM1) is not necessary for p37 to perform this function. Moreover, p14 also has nuclear transport activity, which is different from p37 as p14 only enters the nucleus and is not exported from the nucleus to the cytoplasm $(7,49,50)$. In addition, p10 contains a functional nuclear localization signal (NLS) that can be introduced into the nucleus autonomously in yeast cells and mammalian cells; in the late-stage. p10 protein accumulates in the nucleus suggesting that p10 may perform important functions in the nucleus (51).

ASFV replication mostly depends on its own gene expression. DNA polymerase B, PCNA-like protein, nucleoside triphosphatase and protein fused with DNA primerase encoded by G1211R, E301R, C962R and F1055L respectively, participate in the initiation point replication $(5,7)$. In the early stage of infection, 


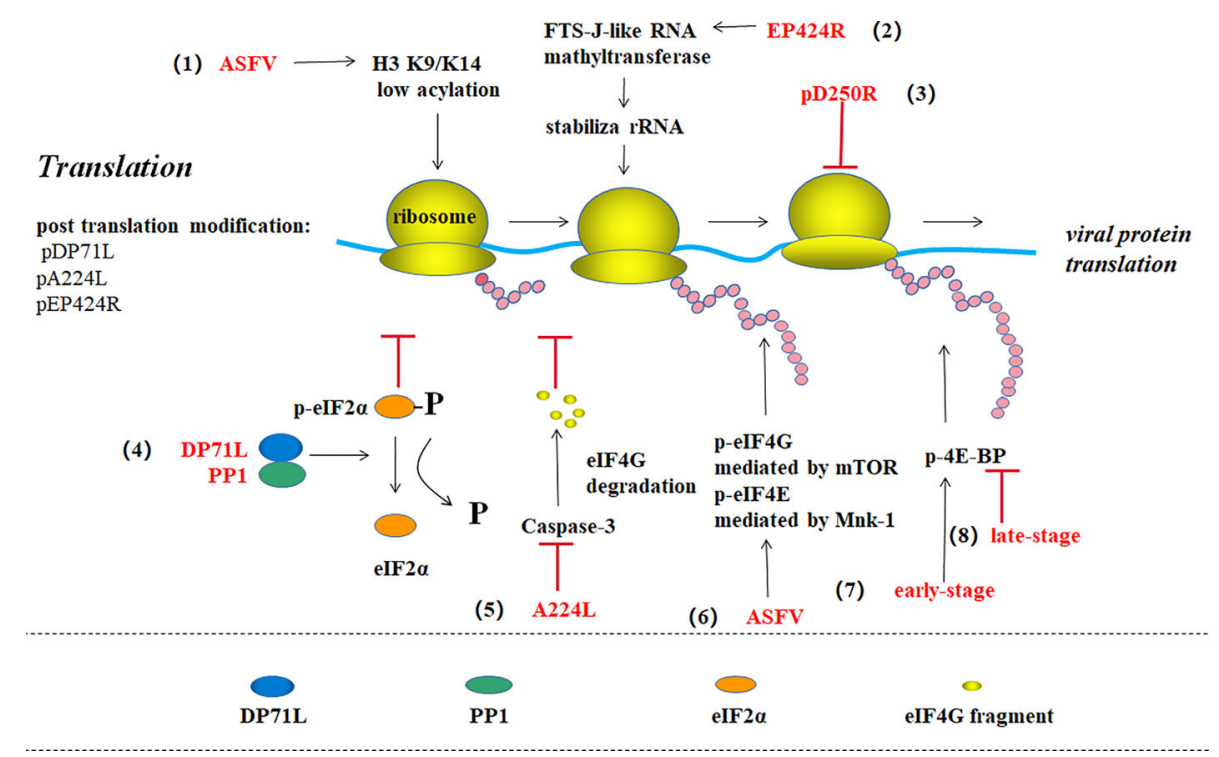

FIGURE 5 | ASFV translation and post-translational modification (39). (1) ASFV promotes viral gene expression by maintaining the host histone H3 K9/K14 in a low acylation state. (2) EP424R-encoded FTS-J-like RNA methyl transferase may stabilize rRNA in cells and prevent protein synthesis shut down. (3) pD250R inhibits viral translation by reducing the number of transcripts. (4) The virus protein DP71L binds to host phosphatase 1 (PP1) to form elF2 alpha dephosphorylation, thus enhancing viral protein synthesis. (5) ASFV IAP homologous protein A224L inhibits the Caspase-3-mediated elF4G degradation to promote translation. (6) ASFV promotes mTOR-mediated elF4G phosphorylation and Mnk-1-mediated elF4E phosphorylation to enhance protein synthesis. (7) ASFV promotes 4E-BP phosphorylation and viral protein translation in the early stage of infection, while (8) promotes 4E-BP dephosphorylation in the late stage to inhibit translation.

ASFV ruptures nucleolus and nuclear membrane, and phosphorylates and then degrades laminin A/C. The resulting membrane fragments are then recruited to the replication site, and nucleoporin p62 is recruited to its periphery. These phenomena may promote viral genome replication (52). In addition, ASFV can also promote $\mathrm{H} 3 \mathrm{~K} 9 \mathrm{me} 3$ (histone $\mathrm{H} 3$ ) and HP1 $\beta$ (heterochromatin protein 1 isoforms) foci formation at early stage with HP1 $\alpha$ and HDAC2 (histone deacetylase 2) enrichment at nuclear. Therefore, ASFV was believed to encourage heterochromatinization of host genome, which may be beneficial for ASFV infection (53). Sulfite sequencing has shown that the nascent ASFV DNA in infected Vero cells is not methylated by the host proteins. As CPG methylation is one of the methods for cells to resist foreign nucleic acids, it has been revealed that ASFV may have a replication mechanism that is at least partially hostindependent (11). The two ASFV-encoded RNA helicases, QP509L and Q706L, are conserved and speculated to be involved in midand late-stage viral gene replication and transcription with nonredundant effects, by detecting their mRNA levels. Recent research reveals that QP509L and Q706L are essential for ASFV producing infectious virus particles (54). The ASFV-encoded topoisomerase II (pP1192R) is transcribed in the early stage and involved in virus replication, transcription, genome agglutination, and separation, which catalyzes double-stranded DNA transient nicking $(3,39)$. However, some other researchers detected pP1192R at intermediate and late phases co-localized with VF (55). It has been found that the purified SFV ORF P1192R-encoded protein in Saccharomyces cerevisiae effectively decomposes kinetoplast DNA (kDNA) and promotes excessive relaxation of supercoiled DNA (7). It is suggested that the activity of PtdIns-converting kinases is necessary for intracellular ASFV replication site establishment, as inhibiting PIKfyve reduces ASFV replication level (33). Furthermore, previous studies have shown that histone-like proteins (pA104R) could bind to ssDNA or dsDNA and cooperate with pE199L to keep DNA-supercoiling activity. Therefore, it is easy to believe that both pA104R and pP1192R play an important role in ASFV replication $(7,32,56,57)$.To avoid the decrease in virus titer due to reactive oxygen-induced DNA damage, ASFV uses the DNA glycosylase of the host cell to repair the damaged DNA through the base excision repair (BER) pathway. The enzymes involved in this process include DNA polymerase type $\mathrm{X}$ (O174L), DNA ligase (NP419L), type II polypurine/cyclic nucleoside endonuclease (pE296R), PCNA-like protein (pE301R), and $5^{\prime}-3^{\prime}$ exonuclease enzyme PD345R (5).

\section{ASFV VF Formation Mechanism}

ASFV hijacks and changes the cellular trans-Golgi endosomal network system to facilitate viral infection. The host adaptor protein AP-1 mediates protein transport from the trans-Golgi network (TGN) to the endosome, recruits clathrin to form clathrin-coated vesicles and selectively carry cargo by recognizing protein sorting signals. AP-1 is recruited to the TNG membrane under the manipulation of small GTPase ADPribosylation factor 1 (Arf1). Intracellular AP-1 localization and clathrin-encapsulated body/endosome migration in the cell induces new membrane structure formation due to the interaction between the outer envelope proteins $C D 2 \mathrm{v}$ and $\mathrm{AP}-1$ (31). Previous studies have reported that various forms and small 
membrane fragments are involved in the early stage of VF formation, which grows into curved membranes and then forms assembly intermediates. These assembly intermediates of the membrane accumulate and aggregate to form a network structure, while others have observed a heliciform structure (38, 58). Together with the viral genome, viral replicase, and host proteins, these membrane structures comprise the center of viral replication and morphogenesis called the VF and is located in the microtubule organizing center (MTOC) (31). The presence of structural proteins p54 and p34 has been observed in the VF membrane structure. Confocal microscopy has shown that $\mathrm{p} 72$ and p54 in the VF are morphologically different. p72 is punctate, while p54 is a polymorphic structure that may be related to the connection between p54 and newly formed virus particles (38). Interestingly, ASFV infection reshapes cholesterol distribution in the cells (31). The intake of cholesterol to cell is a key factor for virus to enter the host cell, replicate and form the VF. On the contrary, cholesterol is not allowed to accumulate in the endosome, otherwise virus particles will be retention in the endosome. A large number of lipid transfer proteins, such as oxysterol binding protein (OSBP), mediate lipid and ion exchange in the VF. Viruses utilize the membrane contact site (MCS) to ensure lipid transfer to the $\operatorname{VF}(3,31,59)$. The virus particles recruit PI4P kinase to form $\mathrm{PI} 4 \mathrm{P}$ aggregates in large numbers, which then recruits OSBP to form a large quantity of MCSs, which accumulate cholesterol through cholesterol and PI4P exchange. In addition, the acylcoenzyme A binding domain containing 3 (ACBD3) required to complete lipid exchange and phosphatidylinositol-phosphate-4kinase III $\beta$ (PI4K $\beta$ ) has been found in the VF of ASFV-infected cells (59). Studies have shown that ASFV-encoded g5Rp has a Nudix hydrolysis motif, which may play a role in regulating virus morphogenesis involving inositol diphosphate polyphosphatemediated membrane transport (60). The ASFV j4R protein interacts with the $\alpha$-chain of nascent polypeptide-associated complex (NAC) which indicating that $\mathrm{j} 4 \mathrm{R}$ may be involved in the transport of newly synthesized proteins to the VF (28).

The complete VF is a single structure lacking an external restriction membrane, and is surrounded by mitochondria and vimentin cage, which provides a physical scaffold for VF or prevents viral components entering the cytoplasm $(33,61)$. VF morphology changes during viral infection. Nevertheless, membrane formation, flow pathway to VF, host cell rearrangement mechanism of host cell due to VF formation, and the relationship between VF and host cell membrane are still unclear (38).

\section{Mature Virus Particle Assembly and Progeny Virus Release Mechanism}

ASFV is assembled at the VF of the host perinuclear region, which is concentrated in the microtube formation center and the Goerki complex (62) (Figure 6). Morphological changes in viral particles occur in the VFs where the core shell proteins of virions are deposited on membrane assembly intermediates to form the core shells (38). A104R, located in the core shell, is a topological isomerase II with high affinity for double-stranded DNA, and thus has been suggested to participate in ASFV genome packing (3). However, pP1192R was thought to participate in genome segregation to facilitate the separation of newly-replicated DNA molecules. B354L and D345L are suggested to involve in processing DNA ends for strand exchange or single-strand annealing during recombination (63). p14, a late-stage nonstructured protein expressed by ASFV 9GL is suppressed, leads to defective interference particles without a central nucleoid (64). The core shell is tightly attached to the inner cystic membrane at first which relies on the $\mathrm{N}$-myristoylation of the viral polyprotein pp220. With viral particle maturation, the core and inner cystic membranes are separated inside the internal capsule. Simultaneously, the virus nucleoid is concentrated, condensed, and merged, which are generally considered to be inseparable from pp220 hydrolysis $(21,38)$. Inhibiting pp220 and pp62 hydrolysis produces non-nuclear or non-infectious viral particles. pS273R, a protein hydrolase, catalyzes the processing of proteases involved in polyprotein precursors. In addition, pB602L and p17 also affects core shell assembly to the inner membrane by affecting pp220 and pp62 hydrolysis (27). After the assembly of the internal capsule is completed, the coat proteins deposited on the membrane assembly intermediate are fitted together one by one making the membrane precursor gradually form an icosahedron structure (62). The packing of the main coat protein p72 requires the assistance of a B602L-encoded molecular chaperone (5). The transmembrane structural protein p54 is essential for ER collection and its transformation into the viral membrane precursor (65). Studies have shown that p54 is antiparallel, exposing cysteine at the N-terminal in the ER, leading to disulfide bond formation-mediated ER collapse (61). Linear array assembly of structural proteins along the ER membrane may also be a cause of ER collapse. Structural proteins assemble on the collapsed ER, forming zipper-like stacking (61). Furthermore, P54 interacts directly with the microtubule dynamic complex by connecting with dynein light chain 8 (LC8) (27). Restraining p54 expression interrupts ER transfer to the virus assembly site, which in turn prevents ER conversion into the membrane precursor, reducing the number of virus offspring (65). With regard to the assembly of icosahedron in the NLDV family, the most popular illustration is the spiral mechanism. In other words, icosahedron assembly starts at the five-fold vertices and is carried out continuously in a spiral manner. Xianjiao et al. believe that the tape measure protein $(\mathrm{TmP})$ of the minor capsid protein ( $\mathrm{mcp}$ ) plays an important role in icosahedra packing. Experiments show that mcp forms a network structure under the major capsid protein (MCP) to stabilize adjacent virocapsomers. Through analysis, $\mathrm{TmP}$ is thought to control capsid size by connecting to the adjacent five-fold vertices and defining the boundaries of trisymmetrons. It is thought to provide a mechanism of assembly from the initial vertices to the adjacent vertices (66). p17 plays a significant role in the transformation of the capsule precursor into icosahedral intermediates. pB602L synthesis inhibition decreases 72 protein and delocalization of the shell protein pEl20R, which produces abnormal "zip-like" structure instead of the icosahedron (67). Inhibiting the expression of the membrane protein $\mathrm{pB} 438 \mathrm{~L}$ results in abnormal tube-like structures instead of an icosahedron (68). Therefore, pB602L 


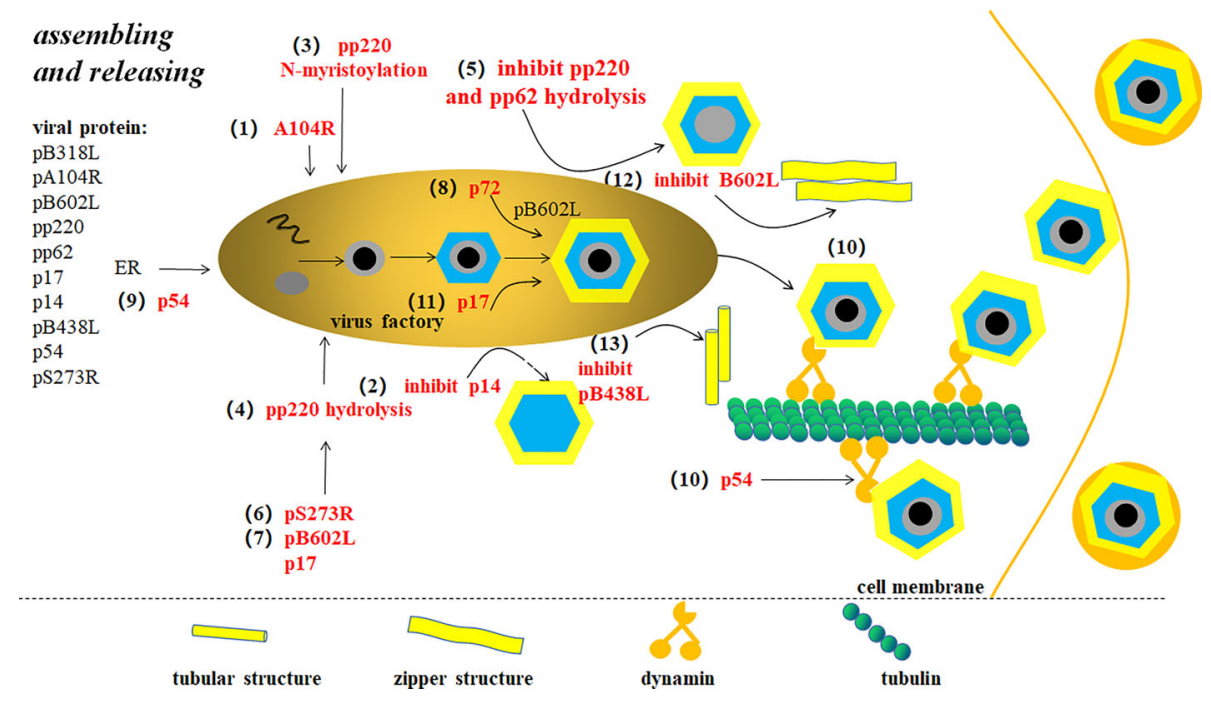

FIGURE 6 | ASFV assembly and release (9). Changes in the virus particle morphology occur in virus factory (VF). (1) A104R is located in the core shell and involved in ASFV genome packaging. (2) p14 expression inhibition produces defective interference particles without a central nucleoid. (3) The core shell adsorbs on the inner membrane depending on pp220 N-bean acrylamide. (4) pp220 hydrolysis separates the core shell from the inner capsule membrane, thus concentrating, condensing, and merging the nucleus. (5) pp220 and pp62 hydrolysis inhibition leads to non-nuclear or non-infectious virus particle production. (6) ASFV proteolytic enzyme pS273R catalyzes protease processing of polyprotein precursors. (7) Proteins that affect pp220 and pp62 hydrolysis include pB602L and p17. (8) The assembly of the major capsid protein p72 requires the assistance of B602L-encoded molecular chaperone. (9) p54 expression inhibition interrupts endoplasmic reticulum (ER) transfer to virus assembly site. (10) P54 proteins interact with microtube power complexes by connecting directly to dynein. Newly-synthesized viruses are linked to kinesins, which drives the virus to move from VF to extracellular. (11) p17 plays an important role in capsule precursor conversion into an icosahedron intermediate. (12) pB602L synthesis inhibition forms abnormal "zip-like" structure instead of icosahedron. (13) Membrane protein pB438L expression inhibition forms abnormal tubular structure instead of icosahedron.

and pB438L play an irreplaceable role in capsid formations. In addition, ASFV-coded trans-pentenyl-transferase (B318L), positioned at the virus assembly site, is associated with the viral membrane precursor from the ER, which may play a role in VF and/or virus assembly (5).

Finally, viruses are transported directly and widely using micro tubes after finishing the assembly, leaving the VF to plasma membrane (33). Mature virus particles leave the VF, pass through the cytoplasm, and are released by the host cells. The interaction between p54 protein and dynamin light chain (LC8) controls the intracellular transport of the microtubule dynamic complex. The direct binding of virus structural proteins with the small molecular dynamic complex indicates the molecular mechanism of microtubule-mediated virus transport. $\mathrm{pEl} 02 \mathrm{R}$ and $\mathrm{pE} 120 \mathrm{R}$ binding with the main shell protein $\mathrm{p} 72$ also have an essential effect on virus transport from the assembly site to the cytomembrane (69). Some studies have shown that newly synthesized viruses move to extramembranous from VF by linking them to kinesin (33).

\section{ASFV IMMUNOMODULATION MECHANISMS}

After ASFV infects porcine macrophages, the hosts first induce an innate immune response and then produce an adaptive immune response. The genome of ASFV is DNA, which can be recognized by cGAS (a kind of pattern recognition receptors (PRRs) of DNA) as pathogen-associated molecular pattern (PAMP), and then activates the expression of type I IFN through cGAs/STING signal pathway. The downstream antiviral genes of type I IFN, such as IFIT family, are also activated and expressed. Type I IFN and interferon stimulated genes (ISGs) can resist ASFV infection. Macrophages and dendritic cells are the main effector cells of natural immune response induced by ASFV infection. Macrophages enable to release a series of cytokines, such as IL-1a, IL-1b and IL-18, and express MHCI to start the adaptive immune response. Plasmacytoid DC (pDC) and natural killer (NK) cells can produce a large amount of interferon to resist ASFV infection. After 8 days of ASFV infection, the antibody level gradually increases, which suggest that there is a humoral immune response in the host. These neutralizing antibodies have a certain protective effect. In addition, cellular immunity, such as $\mathrm{CD} 8 \alpha+\mathrm{T}$ cells, also plays an important antiviral role in ASFV infection. CD4 + CD8 + double positive (DP) T cells can secrete perforin and granzyme, which may also play a role in resisting ASFV infection (70).

However, to survive and produce progeny viruses, the parental virus encodes various proteins to evade the host immune response. This study summarizes the mechanism of ASFV immune evasion and immunosuppression from several aspects, such as the cGAS/STING pathway inhibition, cytokine and chemokine expression regulation, apoptosis and autophagy 
inhibition, adaptive immune response inhibition, and host inflammation regulation (Table $\mathbf{1}$ ).

\section{cGAS/STING Pathway Inhibition}

Several PRRs present in host cells identify pathogenic microorganisms intruding into the cells by recognizing the PAMPs. Host cells initiate natural immune responses by recognizing PAMPs via PRRs. PRRs include TLRs, NLRs, CLRs, RLRs, and cGAS, while PAMPs include endotoxin, peptidoglycan, dsDNA, dsRNA, ssRNA, ssDNA, and unmethylated DNA. After ASFV infects porcine macrophages, the main PRRs are cGAS to recognize the PAMPs, dsDNA, of the virus. cGAS is a cyclic GMP-AMP synthase located in the cytoplasm, which catalyzes the synthesis of cyclic GMP-AMP (cGAMP) after recognizing the viral dsDNA. Subsequently, cGAMP stimulation induces the type I IFN production and activates the transcription factor NF- $\mathrm{KB}$ through the STINGTBK1-IRF3 signaling pathway. IFNs and NF- $\mathrm{KB}$ induce antiviral protein synthesis and proinflammatory factor production through downstream pathways to eliminate the virus.

DP96R of the ASFV China 2018/1 strain is a conservative early expression protein that inhibits the phosphorylation of TBK1 induced by cGAS/STING. DP96R inhibits the activation of IFN- $\beta$ and ISRE promoters by suppressing cGAS/STING and TBK1, but does not inhibit their activation mediated by IRF3-5D. In addition, DP96R can also inhibit the NF- $\kappa \mathrm{B}$ promoter by inhibiting cGAS/STING, TBK1, and IKK $\beta$. The C-side 30-96 aa of DP96R is the active site of its inhibition function (14). Additionally, MGF-505-7R interacted with STING in cells infected with ASFV. MGF-505-7R overexpression promotes autophagy-related protein expression, reduces STING expression, and inhibits the cGAS/STING signaling pathway (15). In addition, Tol-like receptor (including TLR1, TLR2, TLR4, and TLR6) expression decreases in ASFV-infected macrophages (16).

\section{Cytokine Expression Regulation}

Cytokines are active protein molecules secreted by cells that perform signal transduction, participate in immune response and inflammation in different ways, and play an important role in maintaining the normal function of the organism and removing pathogenic microorganisms. Cytokines include interleukins, interferons, TNFs, and chemokines. During ASFV infection, 20 cytokines are significantly upregulated in host cells and four cytokines are downregulated (16). Therefore, it is speculated that ASFV regulates the immune response by manipulating cytokine expression in host cells.

\section{Interferon Induction and Downstream Gene Expression Inhibition}

Interferons are the first line of defense against viral infections and play a considerable role in the early immune response. Type I interferon (IFN- $\alpha / \beta)$ is an important component of innate immunity, and type II interferon (IFN- $\gamma$ ) is a momentous immune response molecule that participates in the entire process of immune response. Furthermore, the combination of interferon with its receptors activates JAK/STAT signal transduction, inducing the expression of several antiviral proteins related to antigen delivery and apoptosis. Therefore, interferon induction suppression seriously disturbs the antiviral reaction of the host.

The ASFV A276R, derived from the MGF360, targets IRF3 instead of IRF7 and IFN- $\beta$ through the cytosolic pathway and TLR3 pathway to suppress IFN- $\beta$ induction. ASFV A528R inhibits IFN- $\beta$ and IRF3 promoter activation in the type I IFN signaling pathway to inhibit type I IFN generation $(15,77)$. ASFV I329L is considered to be a TLR3 homolog because it harbors an extracellular leucine sequence similar to that in TLR3 (78). I329L inhibits activation induction of IFN- $\beta$, NF-B and IRF3 at the TRIF level (77) and the subsequent expression of IFN- $\beta$ and CCL5 (79). Moreover, MGF360 and MGF505/530 inhibit the I-type IFN production and impact (14). ASFV MGF360-12L reduces IFN- $\beta$ and NF- $\kappa B$ promoters activity and inhibits mRNA transcription of IFN- $\beta$, IRF3, STING, TBK1, ISG54, ISG56 and AP-1 (76).

\section{TNF- $\alpha$ Inhibition}

pA238L inhibits TNF- $\alpha$ generation and expression. CBP/p300 is a histone acetyltransferase (HAT) that enables histone acetylation to promote transcription factor-mediated target

TABLE 1 | African swine fever virus (ASFV) regulates inflammation through regulating proinflammatory cytokines, inflammatory mediators, ALR and NF-KB pathway.

\begin{tabular}{|c|c|c|c|}
\hline Regulation pathway & Viral protein & Target molecule & Effect \\
\hline $\begin{array}{l}\text { proinflammatory } \\
\text { cytokines }\end{array}$ & pL83L $(71,72)$ & interact with IL-1 $\beta$ & / \\
\hline inflammatory mediator & pA238L (71, 73-75) & $\begin{array}{l}\text { inhibit p300 } \\
\text { inhibit TNF- } \alpha\end{array}$ & $\begin{array}{l}\text { inhibit NO } \\
\text { inhibit prostaglandin }\end{array}$ \\
\hline ALR & $\begin{array}{l}\text { pS183L, pE199L、p061R、pl7L } \\
(71,75)\end{array}$ & / & Activate AIM2 inflammasome \\
\hline & $\begin{array}{l}\text { pl226L、pA151R、pNP419、pQP383R } \\
\text { (75) }\end{array}$ & / & inhibit AIM2 inflammasome \\
\hline$N F-\kappa B$ & $\begin{array}{l}\text { pA238L }(71,73,74) \\
\text { MGF-360-12L protein (76) }\end{array}$ & $\begin{array}{l}\text { inhibit p300/CBP } \\
\text { binding with p65 } \\
\text { promoting p65 nuclear exporting } \\
\text { inhibit the interaction between p } 65 \text { and importin } \alpha \\
\text { inhibit the interaction between NF- } \mathrm{kB} \text { andnuclear transport } \\
\text { proteins }\end{array}$ & 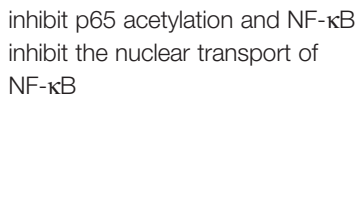 \\
\hline
\end{tabular}


gene transcription. pA238L replaces the combination of cyclic AMP-responsive element/ $/ 3$ complex with $\mathrm{CBP} / \mathrm{p} 300$ on the TNF- $\alpha$ promoter, inhibiting TNF- $\alpha$ activation. In addition, pA238L also inhibits TNF-promoter activation by interfering with the function of $\mathrm{CBP} / \mathrm{p} 300$ by trans-activating transcription factors NF- $\kappa B, N F-A T$, and c-Jun (80). The expression of two signal sensors (FADD and TRADD) of TNF were also decreased $9 \mathrm{~h}$ post ASFV infection (16). TNF- $\alpha$ is a proinflammatory cytokine that triggers an inflammatory response and induces apoptosis through downstream TNF- $\alpha$ signal transduction. In summary, TNF- $\alpha$ generation and expression inhibition is also a mechanism of ASFV immune evasion and immunosuppression.

\section{Interleukin Regulation}

Studies have found that ASFV upregulates immunosuppressive cytokine expression to restrain the host immune response. Interleukin-1 (IL-1), an endogenous pyrogen, causes an inflammatory response and promotes $\mathrm{B}$ cell proliferation and differentiation when combined with IL-1 receptor (IL-1R). In ASFV-infected cells, IL-1R antagonist (IL-1RN), a natural IL-1 antagonist, increases significantly and inhibits the combination of IL-1 with IL-1R, which competitively inhibits the effect of IL-1. In contrast, the expression of IL27, which suppresses the immune response of Th1 and Th17 cells, is downregulated. ASFV L83L encodes a protein that binds to IL-1 $\beta$. However, deletion of this gene does not attenuate ASFV, indicating a redundancy mechanism of ASFV that interferes with IL-1 production (16). Till date, the exact mechanism through which ASFV evades the immune response by regulating host cytokines remains unclear.

\section{Chemokine Regulation}

Chemokines are a class of small proteins that manipulate lymphocyte migration to local inflammation, which assists immune response. ASFV infection significantly induces the chemokine expression to recruit monocytes, T-cells, and natural killer (NK) cells, which are cell-generated antiviral reactions. However, $3 \mathrm{~h}$ post ASFV infection, the expression of chemokines, including CXCL1, CXCL2, CXCL3, and CXCL14, responsible for neutral granulocyte and CD8 T-cell recruitment, was inhibited (16).

\section{Interference With Adaptive Immunity}

The 28-kDa form of A238Lp interacts with cyclophilin and the small subunit of calcineurin, blocking NF-AT activation, thus inhibiting T-cell proliferation and differentiation (17). Furthermore, ASFV EP153R regulates MHC-I expression by interacting with MHC-I through NKG2D and ULBP3, which inhibits MHC-I transport from the ER to the cell membrane instead of affecting MHC-I synthesis or maturation (14). MHC-I decrease may inhibit NK cell activation $(81,82)$. In addition to inhibiting MHC-I, ASFV also prevents the binding of short antigen peptides to MHC-II. MHC-II-coded DMA/DMB removes the class II-associated invariant chain peptide CLIP/ CD74 in the groove to promote the binding of exogenous antigen peptides with MHC-II, while MHC-II-encoded DOA/DOB can inhibit this function. About $9 \mathrm{~h}$ post ASFV infection, SLA-DMA and SLA-DMB expression reduced in swine macrophages, but SLA-DOA and SLA-DOB expression increased. Thus, ASFV has been suggested to inhibit the immune response by preventing antigen binding to MHC-II. In addition, antigens are digested and processed into short peptides before being loaded onto MHC-II mainly by cathepsins. Cathepsin expression in ASFVinfected cells is lower than that in uninfected cells, which inhibits antigen binding to MHC-II. Therefore, ASFV achieves immunosuppression by inhibiting the processing of MHC-II antigens (16). In addition, CD2v inhibits lymphocyte activation in vitro (14).

\section{Apoptosis Inhibition in Host Cells}

There are two common apoptotic pathways: the extrinsic apoptotic pathway (EAP), also known as the death receptor pathway, and the intrinsic apoptotic pathway (IAP), also called the mitochondrial apoptotic pathway. EAP-mediated apoptosis involves TNF family members and death receptors (including FASL/FASR and TNF- $\alpha$ /TNFR1) in the cell membrane. The binding of the death receptor to the ligand produces intracellular apoptosis signals, which then recruits FADD and TRADD as well as caspase 8 to form a death-inducing signaling complex (DISC) and finally activates caspase 8 and caspase 3 successively for apoptosis. However, in IAP-mediated apoptosis, in-cell apoptosis stimulators (e.g., viral proteins) interact with $\mathrm{Bcl}-2$ family apoptosis factors (e.g., Bax, Bak) or anti-apoptotic molecules (e.g., Bcl-2) on the mitochondria, releasing Cyt-c from the mitochondria to the cytoplasm. Cyt-c, APAF-1, and caspase 9 form apoptosomes, which activates caspases 9/3/6/7, thus causing apoptosis. By inducing apoptosis, the host can expose the virus to the immune system and promote virus removal, which is beneficial for maintaining its stability. Hence, to survive, complete replication cycles, and produces more offspring; viruses have evolved an apoptosis-specific immune escape mechanism.

Studies have shown that ASFV A179L, EP153R, DP71L, and A224L suppress ASFV infection-induced apoptosis (14). A179L inhibits both dead receptor-mediated and mitochondrial apoptosis pathways. A179L sequence is very similar to Bcl-2 sequence and considered to be homologous to $\mathrm{Bcl}-2$. A179L is a pan-apoptosis $\mathrm{Bcl}-2$ binding protein $(71,83)$, maintaining high affinity with upstream proteins (Bid, Bim, and Puma) and downstream proteins (Bax and Bak) to ensure its extensive apoptosis inhibition. The interaction between A179L and antiapoptotic proteins is mediated by a domain similar to that of $\mathrm{BH} 3$ encoded by A179L (71). Bid is cut by caspase 8 or granzyme $\mathrm{B}$ produced by cytotoxic $\mathrm{T}$ cells in the process of killing target cells to produce $\mathrm{tBid}-\mathrm{p} 13$, the active form of the bid protein. The BH3 domain-dependent interaction between A179L and tBidp13 inhibits apoptosis (84). However, other studies have concluded that the $\mathrm{BH} 1$ domain in A179L is necessary for the combination of A179L and Bcl-2 (72). pE153R has a C-type lectin domain and inhibits p53-mediated apoptosis and caspase 3 activity. A224L, a member of the inhibitor of IAP-mediated apoptosis family, has a BIR sequence and can inhibit apoptosis under TNF- $\alpha$ induction (71). A224L is involved in multiple ways to suppress apoptosis. It regulates the protease processing of 
caspase 3 by inhibiting caspase 3 protease activity and interacting with the hydrolytic fragments of caspase 3, thus restraining caspase 3 activity (46). It promotes the transcription of many anti-apoptotic genes such as IAP, A20, Bcl-X, Bcl-2, and the IPA family to suppress apoptosis, by activating NF- $\kappa B$. In addition, it also activates cFLIP to inhibit caspase 8 activities $(71,85)$. DP71L inhibits apoptosis by suppressing the unfolded protein response induced by ER stress. DP71L recruits PPI in combination with PPI to dephosphorylate eIF- $2 \alpha$ and inhibit ATF4 and its downstream pro-apoptotic transcription factor CHOP that triggers ER stress-mediated apoptosis. Thus, DP71L inhibits apoptosis by inhibiting CHOP expression $(44,71)$. In addition, the expression of the pro-apoptotic gene GADD45A (growth arrest and DNA-damage-inducible $45 \alpha$ ) was also reduced (16).

\section{Autophagy Inhibition in Host Cells}

Autophagy refers to the biological process of incorporating foreign matters in the cellular autophagosome and cyclic utilization through lysosomal degradation. This process is also known as heterophagy, in which pathogenic microorganisms are incorporated into the autophagosome. Autophagy is important for the elimination of viruses and the maintenance of intracellular environmental stability. Thus, viruses have evolved a series of countermeasures to fight autophagy in cells, which is also an aspect of viral immune escape. ATG2A, ATG9A, ATG101, ATG4B, and ATG7 are autophagy-related genes downregulated in ASFV-infected host cells. However, nuclear protein 1 (NUPR1), which inhibits autophagy, is upregulated during ASFV infection. In addition, BCL2 interacting protein 3 (BNIP3) also induces autophagy and apoptosis and is significantly reduced $9 \mathrm{~h}$ after ASFV infection. It has been speculated that the combination of the A179L and $\mathrm{BH} 3$ domains of Beclin-1 may inhibit autophagy (71). Grooves in the binding region of the A179L spatial configuration are necessary for the combination of A179L and Beclin-1. The ability of the A179L mutant to bind to Beclin-1 decreased the ability of A179L to inhibit autophagy (86).

\section{Inflammation Regulation}

Inflammatory reactions are effective as host antiviral therapies. The host regulates inflammatory responses through proinflammatory factors, inflammatory mediators, and proteins produced in downstream pathways of the NF- $\kappa \mathrm{B}$ and ALR pathways. Once the regulation is unbalanced, an inflammatory factor storm is produced in the organism, causing damage to normal cells. The virus controls cellular inflammation by regulating the production and expression of these substances in favor of viral survival (Table 1).

\section{Inflammatory Mediator Inhibition}

Viral proteins inhibit inflammatory reactions by reducing the production of inflammatory mediators, such as $\mathrm{NO}$ and prostaglandin. pA238L is an effective anti-inflammatory protein that inhibits the inflammatory response of cells. Inducible nitric oxide synthase (iNOS) induces nitric oxide synthase (NOS) to produce NO, and cyclo-oxygenase (COX) catalyzes arachidonic acid conversion into prostaglandin. iNOS transcription is regulated by the co-activation factor, $\mathrm{CBP} / \mathrm{p} 300$. P300 overexpression enhances iNOS promoter activity. However, A238L expression indirectly inhibits the activity of iNOS promoters by inhibiting the effect of p300. Moreover, A238L also inhibits TNF- $\alpha$ activation-induced NOS and COX activation pathway, as well as cox-2 transcription and prostaglandin production.

\section{NF-кB Pathway Inhibition}

In addition to suppressing inflammation by inhibiting NO, prostaglandin, and NOS production, pA238L inhibits inflammation by inhibiting NF- $\kappa \mathrm{B}$. First, A238L suppresses $\mathrm{NF}-\kappa \mathrm{B}$ by inhibiting acetylation of p65. p300/CBP is a histone acetyltransferase (HAT) enzyme, which directly acetylates p65. A238L prevents p300/CBP-mediated p65 acetylation, thus reducing its affinity for DNA binding (71, 73, 74). Secondly, $\mathrm{A} 238 \mathrm{~L}$, an $\mathrm{I} \kappa \mathrm{B} \alpha$ homolog, has an Ankyrin repeat sequence similar to $\mathrm{I} \kappa \mathrm{B} \alpha$. The translation-modified $32-\mathrm{kDa}$ form of A238L directly interacts with the $65 \mathrm{kDa}$ subunit of NF-B to form the A238L-p65 complex located in the cytoplasm, inhibiting p65 entry in the nucleus and the binding of NF- $\kappa B$ to DNA. In addition, unlike $\mathrm{I} \kappa \mathrm{B} \alpha, \mathrm{A} 238 \mathrm{~L}$ also inhibits NF- $\kappa \mathrm{B}$ activity by enhancing NF- $\kappa \mathrm{B}$ export in the nucleus. NF- $\kappa \mathrm{B}$ activity inhibition suppresses of the expression of inflammatory cytokines initiated through the NF- $\kappa B$ signal transduction pathway (17). Besides, MGF360-12L inhibits NLS-mediated intranuclear NF- $\mathrm{KB}$ localization. Karyopherin, comprising importins $\alpha$ and $\beta$, is responsible for NF- $\mathrm{KB}$ localization. The interactions of MGF36012L with KPNA2 (importin $\alpha 2$ ), KPNA3 (importin $\alpha 3$ ), and KPNA4 (importin $\alpha 4$ ) inhibit the interaction between $\mathrm{p} 65$ and importin $\alpha$. Moreover, MGF360-12L inhibits the nuclear transport of NF- $\kappa \mathrm{B}$ by inhibiting the interaction between NF- $\kappa \mathrm{B}$ and nuclear transport proteins (76).

However, some studies have reported opposite results. Rhiannon et al. have found that $\mathrm{A} 238 \mathrm{~L}$ accumulates in the nucleus during the late-stage infection of recombinant ASFV strains. Moreover, A238L expression neither inhibits the nuclear import of NF- $\mathrm{BB}$ p50 or p65 subunit, nor inhibits the CRM1mediated nuclear exit of p65 (73).

Interestingly, A238L is an early protein that inhibits the inflammatory response of cells, while A224L is a late protein in the viral cycle that promotes it. This may indicate that the virus needs low NF- $\kappa \mathrm{B}$ activity at an early stage to avoid an immune response, but high activity at a later stage may prevent apoptosis (85).

\section{ALR Pathway and Inflammation Regulation}

ASFV also regulates cellular inflammation by controlling the ALR pathway. AIM2 inflammasomes are produced after ALR recognizing the dsDNA located in the cytoplasm. Interestingly, four ASFV-encoded proteins, S183L, E199L, O61R, and I7L, activate the AIM2 inflammasome in the ASFV-infected host cell, while four ASFV-encoded proteins, I226L, A151R, NP419L, and QP383R, inhibit AIM2 inflammasome activation (75).

In addition, the combination of pL83L and IL-1 $\beta$ may regulate inflammatory factor activity (71). 


\section{DISCUSSION}

Till date, there are no effective vaccines against ASFV, which makes ASF prevention or control difficult. Therefore, basic research on ASFV is still needed to provide a theoretical basis for vaccine research. On the one hand, the life cycle of ASFV in host cells needs to be further studied. On the other hand, the host immune response to ASFV and the immunosuppressive mechanism of ASFV evolution need a further research.

Although the detailed process of ASFV infected cells has been described in previous studies, the involved proteins of cells and viral are still unknown. It is possible that multiple molecules as receptors or coreceptors are involved in the viral infection process. The viral proteins involved in entry into host cells can be used as targets for ASFV attenuation, which offers possibilities for successful vaccine development. When ASFV successfully enters host cells, it forms a VF near the perinuclear region, which is necessary for newborn virus assembly. VF is a type of membrane; hence VF formation requires lipid accumulation. However, the mechanism of VF membrane structure formation is unclear. Some studies have suggested that VF originates from the ER and intracellular vesicular system, while others have observed viral protein expression collapses the $\mathrm{ER}(5,61)$. In addition, ASFV also replicates within the nucleus, and ASFV DNA entry into the nucleus may be related to viral proteins $\mathrm{p} 37$, p14, and p10 that can enter the nucleus $(7,49-51)$. However, the viral protein-mediated transport of large viral DNA to the nucleus, DNA replication, and DNA transport to cytoplasm should be studied in the future.

As is known to all, the organism produces a series of antiviral responses due to the invasion of ASFV, while ASFV can also form its own immune escape mechanism against the antiviral responses. Aiming at the production of type I interferon through cGASSTING signal pathway, virulent ASFV strain can inhibit the production of type I IFN by regulating some adaptor molecules of this signal pathway, such as regulating the activity of IRF3, the phosphorylation of STING and its translocation in cytoplasm. As for the antiviral effect of macrophages and dendritic cells, ASFV can down regulate the expression of CD14 and CD16 in infected macrophages, which weaken its antiviral and antimicrobial response. Compared with attenuated strain, virulent ASFV strain cannot activate NK cells by down regulating the expression of MHCI in macrophages and dendritic cells, thereby avoiding the recognition of host innate immunity. In addition, the virulent strain may also have the ability, lost by attenuated strain, to avoid the production and release of key cytokines (IFN- $\alpha$, IFN- $\beta$, IL- $1 \beta$ and so on) by macrophages, which weakens the immune surveillance and $\mathrm{T}$ cell response ability of macrophages. In addition, some ASFV genes can also inhibit transcription factors (such as IRF3 and NF$\mathrm{KB}$ ) to inhibit the production of type I IFN in macrophages, such as ASFV multigene families (MGF) 360 and 530/505 and I329l.

\section{REFERENCES}

1. Alejo A, Matamoros T, Guerra M, Andrés G. A Proteomic Atlas of the African Swine Fever Virus Particle. J Virol (2018) 92:e01293-18. doi: 10.1128/JVI.01293-18

2. Revilla Y, Perez-Nunez D, Richt JA. African Swine Fever Virus Biology and Vaccine Approaches. Adv Virus Res (2018) 100:41-74. doi: 10.1016/bs.aivir.2017.10.002
Virulent ASFV strain can also inhibit the expression of ISGS. As for the adaptive immunity produced by host cells, ASFV may inhibit the immune response through regulatory $\mathrm{T}$ cells $(70)$.

However, ASFV genome encodes more than 100 non-structural proteins, most of which are involved in immune response regulation. The mechanisms for immune invasion and immunosuppression by ASFV have been partially elucidated, but it mostly remains unclear. ASFV often inhibits the expression of certain antiviral proteins or immune responses in several ways. For example, A276R and I329L inhibit IFN- $\beta$ expression through different mechanisms, and A179L, A224L, and DP71L inhibit apoptosis. This redundancy may also be one reason for the difficulty in developing vaccines. Therefore, studies on the functions of ASFV-encoded proteins, particularly the proteins mediating ASFV immune escape and immunosuppression, is very important for a live-attenuated vaccine development and design.

In summary, the next work will focus on a comprehensive study and analysis of the function of ASFV protein. We will focus on the proteins involved in the virus life cycle (adsorption, internalization, shelling, biosynthesis, assembly and release) and immunosuppressive function. Because these proteins are most likely the key virulence factors of virus, they are very important for the development of attenuated vaccine. Weakening the virulence gene of virus is one of the strategies to construct attenuated vaccine. For example, the attenuated vaccine of Fowlpox virus was obtained by weakening $(87,88)$. Similarly, an effective vaccine for Ebola virus is developed by replacing the glycoprotein on the surface of recombinant Vesicular Stomatitis Virus with the glycoprotein that can recognize Ebola virus $(89,90)$.

\section{AUTHOR CONTRIBUTIONS}

$\mathrm{YW}, \mathrm{HZ}$ and DL conceived and designed the study. YW, WY, WK, JZ, HZ, and DL wrote the manuscript. All authors contributed to the article and approved the submitted version.

\section{FUNDING}

This work was supported by grants from the Gansu major science and technology projects (20ZD7NA006), National Natural Science Foundation of China (31941002).

\section{ACKNOWLEDGMENTS}

We thank the other members of the HZ lab for their constructive comments.

3. Karger A, Perez-Nunez D, Urquiza J, Hinojar P, Alonso C, Freitas FB, et al. An Update on African Swine Fever Virology. Viruses (2019) 11(9):864. doi: 10.3390/ v11090864

4. YNAU, Microbiology KJ. The Advancement on Molecular Biology of African Swine Fever Virus. Microbiology (2007) 34(3):0572-04. doi: 10.13344/ j.microbiol.china 2007.03.044 
5. Dixon LK, Chapman DA, Netherton CL, Upton C. African Swine Fever Virus Replication and Genomics. Virus Res (2013) 173:3-14. doi: 10.1016/ j.virusres.2012.10.020

6. Quembo CJ, Jori F, Vosloo W, Heath L. Genetic Characterization of African Swine Fever Virus Isolates From Soft Ticks at the Wildlife/Domestic Interface in Mozambique and Identification of a Novel Genotype. Transbound Emerg Dis (2018) 65:420-31. doi: 10.1111/tbed.12700

7. Gaudreault NN, Madden DW, Wilson WC, Trujillo JD, Richt JA. African Swine Fever Virus: An Emerging DNA Arbovirus. Front Vet Sci (2020) 7:215. doi: $10.3389 /$ fvets.2020.00215

8. Sanchez EG, Perez-Nunez D, Revilla Y. Mechanisms of Entry and Endosomal Pathway of African Swine Fever Virus. Vaccines (Basel) (2017) 5(4):42. doi: 10.3390/vaccines5040042

9. Galindo I, Alonso C. African Swine Fever Virus: A Review. Viruses (2017) 9 (5):103. doi: 10.3390/v9050103

10. Cackett G, Matelska D, Sykora M, Portugal R, Malecki M, Bahler J, et al. The African Swine Fever Virus Transcriptome. J Virol (2020) 94:e00119-20. doi: 10.1128/JVI.00119-20

11. Weber S, Hakobyan A, Zakaryan H, Doerfler W. Intracellular African Swine Fever Virus DNA Remains Unmethylated in Infected Vero Cells. Epigenomics (2018) 10:289-99. doi: 10.2217/epi-2017-0131

12. Du X, Gao ZQ, Geng Z, Dong YH, Zhang H. Structure and Biochemical Characteristic of the Methyltransferase (MTase) Domain of RNA Capping Enzyme From African Swine Fever Virus. J Virol (2020) 95(5):e02029-20. doi: 10.1128/JVI.02029-20

13. Frouco G, Freitas FB, Martins C, Ferreira F. Sodium Phenylbutyrate Abrogates African Swine Fever Virus Replication by Disrupting the VirusInduced Hypoacetylation Status of Histone H3K9/K14. Virus Res (2017) 242:24-9. doi: 10.1016/j.virusres.2017.09.009

14. Wang $\mathrm{X}, \mathrm{Wu}$ J, Wu Y, Chen $\mathrm{H}$, Zhang $\mathrm{S}$, Li J, et al. Inhibition of cGASSTING-TBK1 Signaling Pathway by DP96R of ASFV China 2018/1. Biochem Biophys Res Commun (2018) 506:437-43. doi: 10.1016/j.bbrc.2018.10.103

15. Li D, Yang W, Li L, Li P, Ma Z, Zhang J, et al. African Swine Fever Virus MGF505-7r Negatively Regulates cGAS-STING-Mediated Signaling Pathway. J Immunol (2021) 206(8):1844-57. doi: 10.4049/jimmunol.2001110

16. Zhu JJ, Ramanathan P, Bishop EA, O’Donnell V, Gladue DP, Borca MV. Mechanisms of African Swine Fever Virus Pathogenesis and Immune Evasion Inferred From Gene Expression Changes in Infected Swine Macrophages. PLoS One (2019) 14:e0223955. doi: 10.1371/journal.pone.0223955

17. Tait SW, Reid EB, Greaves DR, Wileman TE, Powell PP. Mechanism of Inactivation of NF-Kappa B by a Viral Homologue of I Kappa B Alpha. SignalInduced Release of I Kappa B Alpha Results in Binding of the Viral Homologue to NF-Kappa B. J Biol Chem (2000) 275:34656-64. doi: 10.1074/jbc.M000320200

18. Xian Y, Xiao C. The Structure of ASFV Advances the Fight Against the Disease. Trends Biochem Sci (2020) 45:276-8. doi: 10.1016/j.tibs.2020.01.007

19. Wang N, Zhao D, Wang J, Zhang Y, Wang M, Gao Y, et al. Architecture of African Swine Fever Virus and Implications for Viral Assembly. Science (2019) 366:640-4. doi: 10.1126/science.aaz1439

20. Angulo A, Viñuela E, Alcamí A. Inhibition of African Swine Fever Virus Binding and Infectivity by Purified Recombinant Virus Attachment Protein P12. J Virol (1993) 67:5463-71. doi: 10.1128/jvi.67.9.5463-5471.1993

21. Andres G. African Swine Fever Virus Gets Undressed: New Insights on the Entry Pathway. J Virol (2017) 91:e01906-16. doi: 10.1128/JVI.01906-16

22. Mousavi SA, Malerød L, Berg T, Kjeken R. Clathrin-Dependent Endocytosis. Biochem J (2004) 377:1-16. doi: 10.1042/bj20031000

23. Mercer J, Helenius A. Virus Entry by Macropinocytosis. Nat Cell Biol (2009) 11:510-20. doi: 10.1038/ncb0509-510

24. Mercer J, Schelhaas M, Helenius A. Virus Entry by Endocytosis. Annu Rev Biochem (2010) 79:803-33. doi: 10.1146/annurev-biochem-060208-104626

25. Hernaez B, Guerra M, Salas ML, Andres G. African Swine Fever Virus Undergoes Outer Envelope Disruption, Capsid Disassembly and Inner Envelope Fusion Before Core Release From Multivesicular Endosomes. PLoS Pathog (2016) 12:e1005595. doi: 10.1371/journal.ppat.1005595

26. Lithgow P, Takamatsu H, Werling D, Dixon L, Chapman D. Correlation of Cell Surface Marker Expression With African Swine Fever Virus Infection. Vet Microbiol (2014) 168:413-9. doi: 10.1016/j.vetmic.2013.12.001
27. Jia N, Ou Y, Pejsak Z, Zhang Y, Zhang J. Roles of African Swine Fever Virus Structural Proteins in Viral Infection. J Vet Res (2017) 61:135-43. doi: 10.1515/jvetres-2017-0017

28. Goatley LC, Twigg SR, Miskin JE, Monaghan P, St-Arnaud R, Smith GL, et al. The African Swine Fever Virus Protein J4r Binds to the Alpha Chain of Nascent Polypeptide-Associated Complex. J Virol (2002) 76:9991-9. doi: 10.1128/JVI.76.19.9991-9999.2002

29. Hernaez B, Alonso C. Dynamin- and Clathrin-Dependent Endocytosis in African Swine Fever Virus Entry. J Virol (2010) 84:2100-9. doi: 10.1128/JVI.01557-09

30. Barrado-Gil L, Galindo I, Martinez-Alonso D, Viedma S, Alonso C. The Ubiquitin-Proteasome System Is Required for African Swine Fever Replication. PLoS One (2017) 12:e0189741. doi: 10.1371/journal.pone.0189741

31. Cuesta-Geijo MA, Chiappi M, Galindo I, Barrado-Gil L, Munoz-Moreno R, Carrascosa JL, et al. Cholesterol Flux Is Required for Endosomal Progression of African Swine Fever Virions During the Initial Establishment of Infection. J Virol (2016) 90:1534-43. doi: 10.1128/JVI.02694-15

32. Matamoros T, Alejo A, Rodriguez JM, Hernaez B, Guerra M, Fraile-Ramos A, et al. African Swine Fever Virus Protein Pe199l Mediates Virus Entry by Enabling Membrane Fusion and Core Penetration. mBio (2020) 11:e0078920. doi: $10.1128 / \mathrm{mBio} .00789-20$

33. Cuesta-Geijo MA, Barrado-Gil L, Galindo I, Munoz-Moreno R, Alonso C. Redistribution of Endosomal Membranes to the African Swine Fever Virus Replication Site. Viruses (2017) 9(6):133. doi: 10.3390/v9060133

34. Jouvenet N, Monaghan P, Way M, Wileman T. Transport of African Swine Fever Virus From Assembly Sites to the Plasma Membrane Is Dependent on Microtubules and Conventional Kinesin. J Virol (2004) 78:7990-8001. doi: 10.1128/JVI.78.15.7990-8001.2004

35. Hernaez B, Escribano JM, Alonso C. Visualization of the African Swine Fever Virus Infection in Living Cells by Incorporation Into the Virus Particle of Green Fluorescent Protein-P54 Membrane Protein Chimera. Virology (2006) 350:1-14. doi: 10.1016/j.virol.2006.01.021

36. Alonso C, Miskin J, Hernáez B, Fernandez-Zapatero P, Soto L, Cantó C, et al. African Swine Fever Virus Protein P54 Interacts With the Microtubular Motor Complex Through Direct Binding to Light-Chain Dynein. J Virol (2001) 75:9819-27. doi: 10.1128/JVI.75.20.9819-9827.2001

37. Cackett G, Sykora M, Werner F. Transcriptome View of a Killer: African Swine Fever Virus. Biochem Soc Trans (2020) 48:1569-81. doi: 10.1042/ BST20191108

38. Aicher SM, Monaghan P, Netherton CL, Hawes PC. Unpicking the Secrets of African Swine Fever Viral Replication Sites. Viruses (2021) 13(1):77. doi: $10.3390 / \mathrm{v} 13010077$

39. Freitas FB, Frouco G, Martins C, Leitao A, Ferreira F. In Vitro Inhibition of African Swine Fever Virus-Topoisomerase II Disrupts Viral Replication. Antiviral Res (2016) 134:34-41. doi: 10.1016/j.antiviral.2016.08.021

40. Kinyanyi D, Obiero G, Obiero GFO, Amwayi P, Mwaniki S, Wamalwa M. In Silico Structural and Functional Prediction of African Swine Fever Virus Protein-B263R Reveals Features of a TATA-Binding Protein. PeerJ (2018) 6: e4396. doi: 10.7717/peerj.4396

41. Quintas A, Perez-Nunez D, Sanchez EG, Nogal ML, Hentze MW, Castello A, et al. Characterization of the African Swine Fever Virus Decapping Enzyme During Infection. J Virol (2017) 91(24):e00990-17. doi: 10.1128/JVI.00990-17

42. Freitas FB, Frouco G, Martins C, Ferreira F. African Swine Fever Virus Encodes for an E2-Ubiquitin Conjugating Enzyme That Is Mono- and DiUbiquitinated and Required for Viral Replication Cycle. Sci Rep (2018) 8:3471. doi: 10.1038/s41598-018-21872-2

43. Barrado-Gil L, Del Puerto A, Muñoz-Moreno R, Galindo I, Cuesta-Geijo M, Urquiza J, et al. African Swine Fever Virus Ubiquitin-Conjugating Enzyme Interacts With Host Translation Machinery to Regulate the Host Protein Synthesis. Front Microbiol (2020) 11:622907. doi: 10.3389/fmicb.2020.622907

44. Zhang F, Moon A, Childs K, Goodbourn S, Dixon LK. The African Swine Fever Virus DP71L Protein Recruits the Protein Phosphatase 1 Catalytic Subunit to Dephosphorylate Eif2alpha and Inhibits CHOP Induction But Is Dispensable for These Activities During Virus Infection. J Virol (2010) 84:10681-9. doi: 10.1128/JVI.01027-10

45. Dixon LK, Sanchez-Cordon PJ, Galindo I, Alonso C. Investigations of Proand Anti-Apoptotic Factors Affecting African Swine Fever Virus Replication and Pathogenesis. Viruses (2017) 9(9):241. doi: 10.3390/v9090241 
46. Nogal ML, Gonzalez de Buitrago G, Rodriguez C, Cubelos B, Carrascosa AL, Salas ML, et al. African Swine Fever Virus IAP Homologue Inhibits Caspase Activation and Promotes Cell Survival in Mammalian Cells. J Virol (2001) 75:2535-43. doi: 10.1128/JVI.75.6.2535-2543.2001

47. Castello A, Quintas A, Sanchez EG, Sabina P, Nogal M, Carrasco L, et al. Regulation of Host Translational Machinery by African Swine Fever Virus. PLoS Pathog (2009) 5:e1000562. doi: 10.1371/journal.ppat.1000562

48. Simoes M, Freitas FB, Leitao A, Martins C, Ferreira F. African Swine Fever Virus Replication Events and Cell Nucleus: New Insights and Perspectives. Virus Res (2019) 270:197667. doi: 10.1016/j.virusres.2019.197667

49. Eulalio A, Nunes-Correia I, Salas J, Salas ML, Simoes S, Pedroso de Lima MC. African Swine Fever Virus P37 Structural Protein Is Localized in Nuclear Foci Containing the Viral DNA at Early Post-Infection Times. Virus Res (2007) 130:18-27. doi: 10.1016/j.virusres.2007.05.009

50. Eulalio A, Nunes-Correia I, Carvalho AL, Faro C, Citovsky V, Simoes S, et al. Two African Swine Fever Virus Proteins Derived From a Common Precursor Exhibit Different Nucleocytoplasmic Transport Activities. J Virol (2004) 78:9731-9. doi: 10.1128/JVI.78.18.9731-9739.2004

51. Nunes-Correia I, Rodriguez JM, Eulalio A, Carvalho AL, Citovsky V, Simoes S, et al. African Swine Fever Virus P10 Protein Exhibits Nuclear Import Capacity and Accumulates in the Nucleus During Viral Infection. Vet Microbiol (2008) 130:47-59. doi: 10.1016/j.vetmic.2007.12.010

52. Netherton CL, Wileman TE. African Swine Fever Virus Organelle Rearrangements. Virus Res (2013) 173:76-86. doi: 10.1016/j.virusres.2012.12.014

53. Simões M, Rino J, Pinheiro I, Martins C, Ferreira F. Alterations of Nuclear Architecture and Epigenetic Signatures During African Swine Fever Virus Infection. Viruses (2015) 7:4978-96. doi: 10.3390/v7092858

54. Freitas FB, Frouco G, Martins C, Ferreira FJEM. The QP509L and Q706L Superfamily II RNA Helicases of African Swine Fever Virus Are Required for Viral Replication, Having Non-Redundant Activities. Emerging Microbes Infect (2019) 8:291-302. doi: 10.1080/22221751.2019.1578624

55. Coelho J, Martins C, Ferreira F, Leito AJV. African Swine Fever Virus ORF P1192R Codes for a Functional Type II DNA Topoisomerase. Virology (2015) 474:82-93. doi: 10.1016/j.virol.2014.10.034

56. Coelho J, Ferreira F, Martins C, Leitao A. Functional Characterization and Inhibition of the Type II DNA Topoisomerase Coded by African Swine Fever Virus. Virology (2016) 493:209-16. doi: 10.1016/j.virol.2016.03.023

57. Frouco G, Freitas FB, Coelho J, Leitão A, Martins C, Ferreira F. DNA-Binding Properties of African Swine Fever Virus Pa104r, A Histone-Like Protein Involved in Viral Replication and Transcription. J Virol (2017), (12):e0249816. doi: 10.1128/JVI.02498-16

58. Suarez C, Gutierrez-Berzal J, Andres G, Salas ML, Rodriguez JM. African Swine Fever Virus Protein P17 Is Essential for the Progression of Viral Membrane Precursors Toward Icosahedral Intermediates. J Virol (2010) 84:7484-99. doi: 10.1128/JVI.00600-10

59. Galindo I, Cuesta-Geijo MA, Del Puerto A, Soriano E, Alonso C. Lipid Exchange Factors at Membrane Contact Sites in African Swine Fever Virus Infection. Viruses (2019) 11(3):199. doi: 10.3390/v11030199

60. Cartwright JL, Safrany ST, Dixon LK, Darzynkiewicz E, Stepinski J, Burke R, et al. The G5r (D250) Gene of African Swine Fever Virus Encodes a Nudix Hydrolase That Preferentially Degrades Diphosphoinositol Polyphosphates. J Virol (2002) 76:1415-21. doi: 10.1128/JVI.76.3.1415-1421.2002

61. Windsor M, Hawes P, Monaghan P, Snapp E, Salas ML, Rodriguez JM, et al. Mechanism of Collapse of Endoplasmic Reticulum Cisternae During African Swine Fever Virus Infection. Traffic (2012) 13:30-42. doi: 10.1111/j.1600-0854.2011.01293.x

62. Cobbold C, Brookes SM, Wileman T. Biochemical Requirements of Virus Wrapping by the Endoplasmic Reticulum: Involvement of ATP and Endoplasmic Reticulum Calcium Store During Envelopment of African Swine Fever Virus. J Virol (2000) 74:2151-60. doi: 10.1128/JVI.74.5.2151-2160.2000

63. Urbano AC, Ferreira FJV. Role of the DNA-Binding Protein Pa104r in ASFV Genome Packaging and as a Novel Target for Vaccine and Drug Development. Vaccines (2020) 8(4):585. doi: 10.3390/vaccines8040585

64. Lewis T, Zsak L, Burrage TG, Lu Z, Kutish GF, Neilan JG, et al. An African Swine Fever Virus ERV1-ALR Homologue, 9GL, Affects Virion Maturation and Viral Growth in Macrophages and Viral Virulence in Swine. J Virol (2000) 74:1275-85. doi: 10.1128/JVI.74.3.1275-1285.2000

65. Rodríguez JM, García-Escudero R, Salas ML, Andrés G. African Swine Fever Virus Structural Protein P54 Is Essential for the Recruitment of Envelope
Precursors to Assembly Sites. J Virol (2004) 78:4299-1313. doi: 10.1128/ JVI.78.8.4299-4313.2004

66. Xian Y, Avila R, Pant A, Yang Z, Xiao C. The Role of Tape Measure Protein in Nucleocytoplasmic Large DNA Virus Capsid Assembly. Viral Immunol (2021) 34:41-8. doi: 10.1089/vim.2020.0038

67. Epifano C, Krijnse-Locker J, Salas ML, Rodriguez JM, Salas J. The African Swine Fever Virus Nonstructural Protein Pb6021 Is Required for Formation of the Icosahedral Capsid of the Virus Particle. J Virol (2006) 80:12260-70. doi: 10.1128/JVI.01323-06

68. Epifano C, Krijnse-Locker J, Salas ML, Salas J, Rodriguez JM. Generation of Filamentous Instead of Icosahedral Particles by Repression of African Swine Fever Virus Structural Protein Pb4381. J Virol (2006) 80:11456-66. doi: 10.1128/JVI.01468-06

69. Andrés G, García-Escudero R, Viñuela E, Salas ML, Rodríguez JM. African Swine Fever Virus Structural Protein Pe120r Is Essential for Virus Transport From Assembly Sites to Plasma Membrane But Not for Infectivity. J Virol (2001) 75:6758-68. doi: 10.1128/JVI.75.15.6758-6768.2001

70. Montoya M, Franzoni G, Pérez-Nuñez D, Revilla Y, Galindo I, Alonso C, et al. Immune Responses Against African Swine Fever Virus Infection, Understanding and Combatting African Swine Fever. Wageningen Academic Publishers (2021). pp. 63-85. doi: 10.3920/978-90-8686-910-7_3

71. Dixon LK, Islam M, Nash R, Reis AL. African Swine Fever Virus Evasion of Host Defences. Virus Res (2019) 266:25-33. doi: 10.1016/j.virusres.2019.04.002

72. Revilla Y, Cebrián A, Baixerás E, Martínez C, Viñuela E, Salas ML. Inhibition of Apoptosis by the African Swine Fever Virus Bcl-2 Homologue: Role of the BH1 Domain. Virology (1997) 228:400-4. doi: 10.1006/viro.1996.8395

73. Silk RN, Bowick GC, Abrams CC, Dixon LK. African Swine Fever Virus A238L Inhibitor of NF-kappaB and of Calcineurin Phosphatase Is Imported Actively Into the Nucleus and Exported by a CRM1-Mediated Pathway. J Gen Virol (2007) 88:411-9. doi: 10.1099/vir.0.82358-0

74. Granja AG, Sabina P, Salas ML, Fresno M, Revilla Y. Regulation of Inducible Nitric Oxide Synthase Expression by Viral A238L-Mediated Inhibition of P65/RelA Acetylation and P300 Transactivation. J Virol (2006) 80:10487-96. doi: 10.1128/JVI.00862-06

75. Song J, Li K, Li T, Zhao G, Zhou S, Li H, et al. Screening of PRRSV- and ASFV-Encoded Proteins Involved in the Inflammatory Response Using a Porcine Igluc Reporter. J Virol Methods (2020) 285:113958. doi: 10.1016/ j.jviromet.2020.113958

76. Zhuo Y, Guo Z, Ba T, Zhang C, He L, Zeng C, et al. African Swine Fever Virus MGF360-121 Inhibits Type I Interferon Production by Blocking the Interaction of Importin Alpha and NF-kappaB Signaling Pathway. Virol Sin (2020) 36(2):176-86. doi: 10.1007/s12250-020-00304-4

77. Correia S, Ventura S, Parkhouse RM. Identification and Utility of Innate Immune System Evasion Mechanisms of ASFV. Virus Res (2013) 173:87-100. doi: 10.1016/j.virusres.2012.10.013

78. Henriques ES, Brito RM, Soares H, Ventura S, de Oliveira VL, Parkhouse RM. Modeling of the Toll-Like Receptor 3 and a Putative Toll-Like Receptor 3 Antagonist Encoded by the African Swine Fever Virus. Protein Sci (2011) 20:247-55. doi: 10.1002/pro.554

79. de Oliveira VL, Almeida SC, Soares HR, Crespo A, Marshall-Clarke S, Parkhouse RM. A Novel TLR3 Inhibitor Encoded by African Swine Fever Virus (ASFV). Arch Virol (2011) 156:597-609. doi: 10.1007/s00705-010-0894-7

80. Granja AG, Nogal ML, Hurtado C, Del Aguila C, Carrascosa AL, Salas ML, et al. The Viral Protein A238L Inhibits TNF-Alpha Expression Through a CBP/p300 Transcriptional Coactivators Pathway. J Immunol (2006) 176:45162. doi: 10.4049/jimmunol.176.1.451

81. Franzoni G, Graham SP, Dei Giudici S, Oggiano A. Porcine Dendritic Cells and Viruses: An Update. Viruses (2019) 11(5):445. doi: 10.3390/v11050445

82. Fraczyk M, Wozniakowski G, Kowalczyk A, Bocian L, Kozak E, Niemczuk K, et al. Evolution of African Swine Fever Virus Genes Related to Evasion of Host Immune Response. Vet Microbiol (2016) 193:133-44. doi: 10.1016/j.vetmic.2016.08.018

83. Banjara S, Caria S, Dixon LK, Hinds MG, Kvansakul M. Structural Insight Into African Swine Fever Virus A179L-Mediated Inhibition of Apoptosis. J Virol (2017) 91(6):e02228-16. doi: 10.1128/JVI.02228-16

84. Galindo I, Hernaez B, Diaz-Gil G, Escribano JM, Alonso C. A179L, a Viral Bcl-2 Homologue, Targets the Core Bcl-2 Apoptotic Machinery and Its Upstream BH3 Activators With Selective Binding Restrictions for Bid and Noxa. Virology (2008) 375:561-72. doi: 10.1016/j.virol.2008.01.050 
85. Rodríguez CI, Nogal ML, Carrascosa AL, Salas ML, Fresno M, Revilla Y. African Swine Fever Virus IAP-Like Protein Induces the Activation of Nuclear Factor Kappa B. J Virol (2002) 76:3936-42. doi: 10.1128/JVI.76.8.3936-3942.2002

86. Banjara S, Shimmon GL, Dixon LK, Netherton CL, Hinds MG, Kvansakul M. Crystal Structure of African Swine Fever Virus A179L With the Autophagy Regulator Beclin. Viruses (2019) 11(9):789. doi: 10.3390/v11090789

87. Giotis ES, Skinner MA. Spotlight on Avian Pathology: Fowlpox Virus. Avian Pathol (2019) 48:87-90. doi: 10.1080/03079457.2018.1554893

88. Bhanuprakash V, Hosamani M, Venkatesan G, Balamurugan V, Yogisharadhya R, Singh RK. Animal Poxvirus Vaccines: A Comprehensive Review. Expert Rev Vaccines (2012) 11:1355-74. doi: 10.1586/erv.12.116

89. Marzi A, Mire CE. Current Ebola Virus Vaccine Progress. Biodrugs (2019) 33:9-14. doi: 10.1007/s40259-018-0329-7

90. Baseler L, Chertow DS, Johnson KM, Feldmann H, Morens DM. The Pathogenesis of Ebola Virus Disease. Annu Rev Pathol (2017) 12:387-418. doi: 10.1146/annurev-pathol-052016-100506
Conflict of Interest: The authors declare that the research was conducted in the absence of any commercial or financial relationships that could be construed as a potential conflict of interest.

Publisher's Note: All claims expressed in this article are solely those of the authors and do not necessarily represent those of their affiliated organizations, or those of the publisher, the editors and the reviewers. Any product that may be evaluated in this article, or claim that may be made by its manufacturer, is not guaranteed or endorsed by the publisher.

Copyright (c) 2021 Wang, Kang, Yang, Zhang, Li and Zheng. This is an open-access article distributed under the terms of the Creative Commons Attribution License (CC BY). The use, distribution or reproduction in other forums is permitted, provided the original author(s) and the copyright owner(s) are credited and that the original publication in this journal is cited, in accordance with accepted academic practice. No use, distribution or reproduction is permitted which does not comply with these terms. 